\title{
ON THE COMPLEX ROOTS OF ALGEBRAIC EQUATIONS*
}

\author{
BY A. J. KEMPNER
}

1. Introduction. The topic I have chosen is so vast that a systematic treatment in an address is impossible. I shall, therefore, restrict my report to some phases of the problem to which I have made contributions, and to such papers as deal with related questions and which have come to my notice.

I sincerely appreciate this opportunity to bring together the results of several articles, all dealing more or less directly with the problem of complex roots, but scattered in various journals over a period of many years.

Our equations are always polynomials, equated to zero, and, unless otherwise stated, with real coefficients. The notation $f_{n}(z)=0$ indicates that the equation is of degree $n$. Merely to answer the question whether equations of high degree are ever actually solved, it may be pointed out that a table of natural sines and cosines, say for every $1^{\prime \prime}$, is nothing but a complete tabulation of the solutions of the equation $x^{1296000}-1=0$.

2. On Equations with Roots $e^{i \theta}$. We have in the literature many theorems on equations with roots of absolute value unity. One of the most interesting of these is Kronecker's theorem:

If the coefficients of an equation are ordinary integers, if the coefficient of the highest power is unity, and if all roots are of absolute value unity, then all the roots are roots of unity, and the equation is therefore solvable by radicals.

A companion theorem is as follows.

With the same restrictions on the coefficients, if all roots are real and of absolute value $<2$, the roots are all of the form $2 \cos \pi k$, where $k$ is rational.

In two short notes $\nmid$ I have derived necessary and sufficient conditions that an equation have some, or all, of its roots of the form $e^{i \theta}$.

* An address presented to the Society, by invitation of the program committee, Lincoln, Nebraska, November 30, 1934.

† Kempner, Archiv der Mathematik und Physik, (3), vol. 25 (1916), pp. 236-242. Kempner, Tôhoku Mathematical Journal, vol. 10 (1916), pp. 115117. 
THEOREM 1. To test any equation $f_{n}(z)=0$ with real coefficients for roots of the form $e^{i \theta}$, form the following polynomial, which has real coefficients and which contains only even powers of $z$ :

$$
\phi_{2 n}(z)=\left(z^{2}+1\right)^{n} \cdot f\left(\frac{z+i}{z-i}\right) \cdot f\left(\frac{z-i}{z+i}\right)=\psi_{n}\left(z^{2}\right)=\psi_{n}(u) .
$$

The equation $f_{n}(z)=0$ has among its roots some of the form $e^{i \theta}$ when and only when $\psi_{n}(u)=0$ has some positive roots. A necessary and sufficient condition that all roots of $f_{n}(z)=0$ be of the form $e^{i \theta}$ is that $\psi_{n}(u)=0$ have only positive roots.

For proof we have only to remember that $z=\left(z_{1}+i\right) /\left(z_{1}-i\right)$ transforms the unit circle in the $z$ plane into the axis of reals in the $z_{1}$ plane. The theorem may be extended in an obvious fashion to equations with complex coefficients, by considering $f(z) \cdot \overline{f(z)}=F(z)$ in place of $f(z)$. It is clear that the theorem applies to much wider classes of functions than polynomials. If $\psi_{n}(u)=0$ has only real positive roots, and if, in addition, the coefficients of $f_{n}(z)=0$ are real integers and the coefficient of the highest power is unity, the roots of $f_{n}(z)=0$ are roots of unity; and, by Kronecker's theorem, the equation $f_{n}(z)=0$ is solvable by radicals.

Making use of the inverse transformation $z_{1}=i(z+1) /(z-1)$ and retracing our steps, we now start from a given equation $\phi_{n}(z)=a_{0} z^{n}+a_{1} z^{n-1}+\cdots+a_{n}=0$ whose roots are all real, and form the new polynomial

$$
\psi_{2 n}(z)=(z-1)^{2 n} \phi_{n}\left(i \frac{z+1}{z-1}\right) \phi_{n}\left(-i \frac{z+1}{z-1}\right)
$$

If $\phi_{n}(z)$ has integral coefficients, the coefficients of $\psi_{2 n}(z)$ are also integers. It is easily seen that the coefficient of $z^{2 n}$ in $\psi_{2 n}(z)$ is $\left(a_{0}-a_{2}+a_{4}-+\cdots\right)^{2}+\left(a_{1}-a_{3}+a_{5}-+\cdots\right)^{2}=\gamma$, say. If $\gamma=1, \psi_{2 n}(z)=0$ satisfies the conditions of Kronecker's theorem. Hence $\psi_{2 n}(z)=0$ is solvable by radicals, and hence also $\phi_{n}(z)=0$ is solvable by radicals.

THEOREM 2. Suppose that $\phi_{n}(z)=a_{0} z^{n}+a_{1} z^{n-1}+\cdots+a_{n}=0$ has real integral coefficients and that all its roots are real. If 
$\left(a_{0}-a_{2}+a_{4}-+\cdots\right)^{2}+\left(a_{1}-a_{3}+a_{5}-+\cdots\right)^{2}=1$, the equation is solvable by radicals.*

3. On Equations with Roots $\alpha+\rho e^{i \theta}$, where $\alpha$ and $\rho$ are Rational. Of greater interest is the investigation of irreducible equations admitting roots of the form $\alpha+\rho e^{i \theta}$, where $\alpha$ and $\rho$ are both rational. $\dagger$ Hand in hand with these equations go those admitting roots of the form $\alpha+\rho e^{i \theta}$, where $\alpha$ and $\rho^{2}$ are rational.

We first characterize geometrically the set of points in the plane of complex numbers corresponding to the set of numbers $\alpha+\rho e^{i \theta}$, where $\alpha$ and $\rho$ are rational. The numbers are represented by the doubly infinite set of circles whose centers are at rational points on the axis of reals, and whose radii are rational. We shall call this set of circles, or the corresponding set of numbers, the set $S$.

Similarly, we shall represent the set of numbers $\alpha+\rho e^{i \theta}$, where $\alpha$ and $\rho^{2}$ are rational, by a corresponding doubly infinite set of circles, and we shall call this set of circles, or the set of numbers, the set $C$.

We shall consider the class of irreducible equations that have any complex roots on any circle $S$. For simplicity, unless otherwise stated, we shall assume that the coefficients are integral and that the equation is irreducible in the natural domain. This class consists of :

(1) all irreducible equations $f(z)=0$ that have any (not necessarily all) complex roots of absolute value 1 ;

(2) all equations derived from (1) by subjecting $f(z)$ to a nonsingular linear transformation $z=\left(a z_{1}+b\right) /\left(c z_{1}+d\right)$ whose coefficients are real and rational.

In particular, all irreducible quadratic equations with rational coefficients and negative discriminant belong to this class. The case (1) contains as a subclass all cyclotomic equations of prime degree.

It turns out that if an irreducible equation with rational coefficients has any root on a circle $S$, or $C$, the roots are sym-

* A systematic examination of applications of Kronecker's theorem may seem desirable. Compare also D. H. Lehmer's article referred to at the end of the next section.

$\dagger$ Kempner, On irreducible equations admitting roots of the form $\alpha+\rho e^{i \theta}, \ldots$, Tôhoku Mathematical Journal, vol. 13 (1918), pp. 253-265. 
metrically distributed with respect to this circle. In this statement, two points are called symmetric with respect to a circle when each is the image of the other under transformation by reciprocal radii. This represents a natural extension of the symmetry property of the axis of reals for all equations with real coefficients.

THEOREM 3. When an irreducible equation has a complex root of the form $\alpha+\rho e^{i \theta}$, where $\alpha$ and $\rho^{2}$ are rational and where $\rho$ is positive, but may be either rational or irrational, the roots of the equation are distributed in the following manner:

(1) Besides $\alpha+\rho e^{ \pm i \theta}$ there may be other pairs of complex roots with the same $\alpha$ and the same $\rho$, that is, of the form $\alpha+\rho e^{ \pm i \theta_{1}}$.

(2) If $\sigma$ is a real root, $\rho^{2} / \sigma$ is a root.

(3) Any complex root not contained in (1) may, of course, be written in the form $\alpha+r e^{i \phi}$, with the same $\alpha$. Then the four numbers $\alpha+r e^{ \pm i \phi}, \alpha+\left(\rho^{2} / r\right) e^{ \pm i \phi}$ are all contained among the roots of the equation.

The complete extension of the symmetry property of the axis of reals, insofar as I can see, may be stated as follows. Let $f(z)=z^{n}+a_{1} z^{n-1}+\cdots+a_{n}=0$ be an equation with real, but otherwise arbitrary, coefficients, irreducible in the domain $R\left(a_{1}, a_{2}, \cdots, a_{n}\right)$, and let us assume that $f(z)=0$ has a root of the form $\alpha+\rho e^{i \theta}$, where $\alpha$ and $\rho^{2}$ are both rationally expressible in terms of $a_{1}, \cdots, a_{n}$ with rational numerical coefficients. Then all roots of $f(z)=0$ are distributed in the plane of complex numbers symmetrically to the axis of reals and to the circle $\alpha+\rho e^{i \theta}$. We may state also the following theorems.

THEOREM 4. When an irreducible equation (in $R(1)$ ) with real coefficients has any complex root on any circle $C$, or on any straight line parallel to the axis of imaginaries and at a rational distance from it, the roots are symmetrically distributed with respect to this circle or straight line.

THEOREM 5. The points of intersection of two circles $S$, which form a set that is everywhere dense in the complex plane, are exactly the set of roots of the class of irreducible equations $a z^{2}+b z+c=0$, where $a, b$, and $c$ are rational and $b^{2}-4 a c<0$. In other words, the set of points of intersection of $S$ are exactly the set of all complex 
irrationalities. The real quadratic irrationalities are given by the points of the axis of reals belonging to $C$, but not to $S$.

It is easily shown that the complex (not real) points of the set of intersections of circles of the set $C$ are the same as the complex (not real) points of the set of intersections of the set $S$. Through each point of intersection of two circles $S$ (or $C$ ) pass an infinite number of circles $S$ (or $C$ ).

Therefore, any imaginary quadratic irrationality may be represented in an infinite number of ways by the form $\alpha+\rho e^{i \theta}$, where $\alpha$ and $\rho$ are rational, but any root of an irreducible equation of degree $n>2$ can be represented in at most one way in this form. Consequently, for $n>2$, the equation $\alpha_{1}+\rho_{1} e^{i \theta_{1}}$ $=\alpha_{2}+\rho_{2} e^{i \theta_{2}}$, where $\alpha_{1}, \alpha_{2}, \rho_{1}$, and $\rho_{2}$ are rational and where $\rho_{1}$ and $\rho_{2}$ are positive, implies that $\alpha_{1}=\alpha_{2}$ and that $\rho_{1}=\rho_{2}$.

It follows from the preceding theorems that if an irreducible equation of degree $n>2$ has roots on a circle $S$, these roots cannot be among the points of intersection of circles $S$.

THEOREM 6. An irreducible equation cannot have roots on more than one circle $S$ unless the circles intersect. On every circle $S$ lie roots of irreducible equations of degree $n>2$, some of whose other roots lie on intersecting circles.

THEOREM 7. An irreducible equation $f(z)=0$ can have two roots $\alpha_{1}+\rho_{1} e^{i \theta_{1}}, \alpha_{2}+\rho_{2} e^{i \theta_{2}}$, where $\alpha_{1}, \alpha_{2}, \rho_{1}$, and $\rho_{2}$ are all rational and $\alpha_{1} \neq \alpha_{2}$, only when $\left|\rho_{1}-\rho_{2}\right|<\left|\alpha_{1}-\alpha_{2}\right|<\rho_{1}+\rho_{2}$.

THEOREM 8. When an irreducible equation $f(z)=0$ has complex roots of the form $z=\alpha+\rho e^{i \theta}$, where $\alpha$ and $\rho$ are rational, $\alpha$ and $\rho$ may be found by a finite number of rational operations and the extraction of a square root. Therefore, if such an equation has a root on any circle $S$, this circle, but not necessarily the root itself, can be constructed by ruler and compasses.

Let us give a numerical illustration. The irreducible equation $16 z^{4}+72 z^{3}+96 z^{2}+60 z+15=0$ is found to have roots of the form $\alpha+\rho e^{i \theta}$, where $\alpha=-1 / 2, \rho=1 / 2$. The equation has two real roots, $\left(-(21)^{1 / 2}-9 \pm\left[30+10(21)^{1 / 2}\right]^{1 / 2}\right) / 8$, and on the circle $-1 / 2$ $+e^{i \theta} / 2$, two complex roots, $\left\{(21)^{1 / 2}-9 \pm\left[30-10(21)^{1 / 2}\right]^{1 / 2}\right\} / 8$.

Necessary and sufficient conditions for an irreducible equation to have complex roots of the form $\alpha+\rho e^{i \theta}$, where $\alpha$ and $\rho$ 
are both rational, are obtained by extending our theorem on necessary and sufficient conditions that an equation have roots of the form $e^{i \theta}$.

TheOREM 9. An irreducible equation $f_{n}(z)=0$ has among its complex roots some roots of the form $\alpha+\rho e^{i \theta}$, where $\alpha$ and $\rho$ are rational, when and only when both of the following conditions are satisfied.

1. The system of equations

$$
\rho^{n} a_{0}=f(\alpha), \quad \rho^{n-2}\left(n a_{0} \alpha+a_{1}\right)=f^{\prime}(\alpha)
$$

has a rational solution $(\alpha, \rho)$.

2. Letting $z=\alpha+\rho z$ and forming the expressions

$$
\begin{aligned}
g\left(z_{1}\right) & =f\left(\alpha+\rho z_{1}\right), \\
\phi_{2 n}\left(z_{1}\right) & =\left(z_{1}^{2}+1\right)^{n} g\left(\frac{z_{1}+i}{z_{1}-i}\right) g\left(\frac{z_{1}-i}{z_{1}+i}\right)=\psi_{n}\left(z_{1}^{2}\right)=\psi_{n}(u),
\end{aligned}
$$

we see that the equation $\psi_{n}(u)=0$ must admit at least one positive root.

As applications, we shall mention the following cases.

(1) Klein's famous Modul-figur, consists, in the plane of complex numbers, of the set of circles of radius $1 / 2$ with centers on the axis of reals at $0, \pm 1, \pm 2, \cdots$, and the system of straight lines parallel to the axes of imaginaries which are tangent to these circles and the system obtained by continued reflection of this figure with respect to itself by reciprocal radii. We may state the following property. An irreducible equation cannot have roots on more than one circle of the Klein Modul-figur.

(2) Every irreducible equation of degree $<10$ with a root on any circle $S$ is completely solvable by radicals.*

(3) When an irreducible equation $a_{0} z^{n}+a_{1} z^{n-1}+\cdots+a_{n}=0$ has a complex root of the form $\alpha+\rho e^{i \theta}$, where $\alpha$ and $\rho$ are rational, the equation $a_{0} y^{n}=a_{0} x^{n}+a_{1} x^{n-1}+\cdots+a_{n}$ has a rational solution $(x, y)$.

When $\alpha$ and $\rho$ are both integers, the diophantine equation $a_{0} y^{n}=a_{0} x^{n}+\cdots+a_{n}$ has an integral solution $(x, y)$.

If the equation $a_{0} z^{n}+a_{1} z^{n-1}+\cdots+a_{n}=0$ has a complex root

* This can probably be extended to give a result corresponding to the last theorem of $\$ 2$. 
on a circle of rational radius touching the axis of imaginaries at the origin, the equation $a_{1} x^{n-1}+a_{2} x^{n-2}+\cdots+a_{n}=0$ has a rational root. The equation $16 z^{4}+72 z^{3}+96 z^{2}+60 z+15=0$, which was mentioned above, comes under this heading. The equation $72 x^{3}+96 x^{2}+60 x+15=0$ has a root $-1 / 2$.

In connection with this work I may mention a paper by $\mathrm{D}$. $\mathrm{H}$. Lehmer. ${ }^{*}$ He defines a polynomial as quasi-cyclotomic if all zeros lie on a circle of radius $r$, with center at the origin, and if the arguments of the roots are commensurable with $2 \pi$. His work is connected with our own by his Theorem 3 , which is as follows.

Let the roots of $f=0$ lie on the circle of radius $r$. Then $r$ is an integer or the square root of an integer, according as $n$ is odd or even.

An interesting result is his Theorem 6, which reads: Let the $n=2 k$ roots of $f(x)=0$ be of the form $(R)^{1 / 2} \cdot \epsilon_{\nu}$, where $\epsilon_{\nu}$ are roots of unity, and where $R$ has no square factor. Then

$$
(R)^{1 / 2} \leqq \frac{(2 k) !}{(k+1) !(k-1) !} .
$$

4. On the Distribution of Complex Algebraic Integers of a Given Degree. The identification of the points of intersection of the set of circles $S$ (or $C$ ) with the totality of complex quadratic irrationalities (integral or otherwise) suggests the problem of investigating the distribution in the plane of complex numbers of the totality of algebraic integers $x+i y$ of a given degree $n=2$, or $n=3$, and so on.

The problem appears artificial on account of the fact that the totalities considered do not form domains of rationality. Yet, it seems to possess a degree of interest. For $n=2$, the solution is given practically by inspection. In the $(x+i y)$ plane, these algebraic integers are obviously distributed along the lines $x=k / 2,(k=0, \pm 1, \pm 2, \cdots)$. The distribution is the same for all lines $x=0, \pm 1, \pm 2, \cdots$, and is also the same for all lines $x= \pm 1 / 2, \pm 3 / 2, \cdots$, but it differs in the two sets. The density increases with increasing $|y|$, and so on. In addition, the integral real quadratic irrationalities are of course distributed everywhere densely on the axis of reals.

For the set of all cubic algebraic integers (and eo ipso those

*Quasi-cyclotomic polynomials, American Mathematical Monthly, vol. 39 (1932), pp. 383-389. 
of higher order), the first suspicion is that they form in the plane of complex numbers an everywhere dense set. Even if this should be so, there is some indication that an examination of the fine structure of the distribution with respect to "gleichmässige Dichtigkeit" may be of interest.

5. On the Use of the Arguments of the Coefficients for Separating Complex Roots. In a previous paper, ${ }^{*}$ I emphasized the possibility of using systematically the arguments of the coefficients of an algebraic equation for the separation of the complex roots. Descartes' rule for an upper bound of positive (negative) roots is the first, very special, case of theorems of this kind. I used a very obvious, yet rather powerful, tool. Consider the equation

$$
\begin{gathered}
f(z)=a_{n} z^{n}+\cdots+a_{0}=0, \\
a_{\mu}=r_{\mu} e^{i \theta_{\mu}}, 0 \leqq \theta_{\mu}<2 \pi, r_{\mu}>0 ; z=\rho e^{i \phi}, 0 \leqq \phi<2 \pi, \rho>0 .
\end{gathered}
$$

In the plane of complex numbers, mark from the origin the half-rays or vectors $\theta_{\mu}$. The vector $\theta_{0}$ is to remain in its original position. The vector $\theta_{1}$ is to rotate in a positive sense with a constant angular velocity $\omega$, while for $\mu=1,2, \cdots, n$, the vector $\theta_{\mu}$ rotates with a uniform angular velocity $\mu$ times that of $\theta_{1}$. Vectors $\theta_{\mu}$ for which $r_{\mu}=0$, that is, for which the coefficient $a_{\mu}=0$, are to be ignored. At any moment the vectors give the directions of the vectors representing the terms $a_{\mu} z^{\mu}=r_{\mu} \rho^{\mu} e^{i\left(\theta_{\mu}+\mu \phi\right)}$. These directions depend only on $\phi$ and the $\theta_{\mu}$ of the coefficients. Most of the theorems are immediate consequences of the fact that the sum of vectors from a common point can certainly not vanish when it is possible to draw a line through the point such that all vectors lie on one side of the line. This fact is used, for example, in the simplest proof of the Gauss-Lucas theorem that all roots of $f^{\prime}(z)=0$ lie inside or on the boundary of the smallest convex polygon around the points representing the roots of $f(z)=0$.

It is, therefore, impossible to have roots of $f(z)=0$ in any sector (vertex at the origin) $\alpha<\phi<\beta$ for which all vectors lie on one side of a straight line through the origin. Such $\phi$-intervals-if they exist-are determined by simple inequalities,

* Kempner, Über die Separation komplexer Wurzeln, Hilbert Festschrift, 1922, pp. 49-59 (= Mathematische Annalen, vol. 85 (1922), pp. 49-59). 
which in many cases enable us to determine sectors, depending only on the arguments of the coefficients, not on the absolute values, and which are free of roots.

The efficiency of the method is a function of the number of coefficients different from 0 , rather than of the degree of the equation. For trinomial equations, a complete separation of the roots is given; for quadrinomial equations, and $m$-nomial equations, $m>4$, valuable information usually is obtained, even though complete separation may not be accomplished.

The results are indicated by the following illustrations.

(1) Binomial equations: $a_{n} z^{n}-a_{0}=0$. We obtain $n$ null sectors (half-rays), each of which contains exactly one root, in agreement with elementary algebra.

(2) Cubic equations: $z^{3}+a z+b=0$, where $a$ and $b$ are real. The three roots have arguments $\phi_{1}, \phi_{2}, \phi_{3}(\phi=0$, real positive roots; $\phi=\pi$, real negative roots);

$$
\begin{array}{lll}
a>0, \quad b>0: & \phi_{1}=\pi, \quad \frac{\pi}{3}<\phi_{2}<\frac{\pi}{2}, & \frac{3 \pi}{2}<\phi_{3}<\frac{5 \pi}{3} ; \\
a>0, \quad b<0: & \phi_{1}=0, \quad \frac{\pi}{2}<\phi_{2}<\frac{2 \pi}{3}, & \frac{4 \pi}{3}<\phi_{3}<\frac{3 \pi}{2} ; \\
a<0, \quad b>0: & \phi_{1}=\pi, \quad 0 \leqq \phi_{2}<\frac{\pi}{3}, & \frac{5 \pi}{3}<\phi_{3} \leqq 2 \pi ; \\
a<0, & b<0: \quad \phi_{1}=0, \quad \frac{2 \pi}{3}<\phi_{2} \leqq \pi, & \pi \leqq \phi_{3}<\frac{4 \pi}{3} .
\end{array}
$$

(3) Trinomial equations: $a_{n} z^{n}+a_{m} z^{m}+a_{0}=0, \quad a_{n}=r_{n} e^{i \theta_{n}}$, $a_{m}=r_{m} e^{i \theta_{m}}, a_{0}=r_{0} e^{i \theta_{0}}$. For such equations, we obtain complete separation of roots, one in each of $n$ sectors.* Special precautions are required for dealing with the cases in which two sectors adjoin and the roots lie on the common boundary line. As an illustration, consider equations of the form

$$
a_{5} z^{5}+a_{2} z^{2}+a_{0}=0, \quad \theta_{5}=0, \quad \theta_{2}=2 \pi / 5, \quad \theta_{0}=5 \pi / 6 .
$$

Each of the following five sectors contains exactly one root: $66^{\circ}<\phi_{1}<84^{\circ}, 129^{\circ}<\phi_{2}<138^{\circ}, 204^{\circ}<\phi_{3}<210^{\circ}, 282^{\circ}<\phi_{4}<309^{\circ}$,

* Partly overlapping results by Nekrassoff, Mathematische Annalen, vol. 29 (1887), pp. 413-440, who has different aims and uses entirely different methods. 
$324^{\circ}<\phi_{5}<354^{\circ}$. The openings of the sectors are, respectively, $18^{\circ}, 9^{\circ}, 6^{\circ}, 27^{\circ}, 30^{\circ}$. This result holds, of course, independently of the absolute values of the coefficients.

(4) Quadrinomial equations. As an illustration, consider any equation of the form

$$
a_{10} z^{10}+a_{9} z^{9}+a_{5} z^{5}+a_{0}=0, \quad\left(a_{0}, a_{5}, a_{9}, a_{10}>0\right) .
$$

Roots are separated into ten sectors: $18^{\circ}<\phi_{1}<36^{\circ}, 36^{\circ}<\phi_{2}$ $<60^{\circ}, 90^{\circ}<\phi_{3}<108^{\circ}, 108^{\circ}<\phi_{4}<140^{\circ}, 162^{\circ}<\phi_{5} \leqq 180,180^{\circ}$ $\leqq \phi_{6}<198^{\circ}, \cdots, 324^{\circ}<\phi_{10}<342^{\circ}$. The largest opening is $32^{\circ}$, the smallest $18^{\circ}$. Complete separation is partly due to the high degree of the equation, despite the small number of terms. For quadrinomial equations, complete separation is no longer guaranteed.

(5) Mechanical Determination of Sectors. An instrument of the type of a "planetarium" which permits $n$ vectors to rotate from arbitrary initial positions with angular velocities which are to each other in the ratios $n: n-1: \cdots: 3: 2: 1$ would permit the mechanical determination of sectors free from roots. An ordinary watch will separate all roots of any equation of the form

$$
z^{12} \pm a z^{\lambda} \pm b=0, \quad(a>0, b>0, \lambda=1 \text { or } 11) .
$$

Consider, for example, any equation of the form $z^{12}+a_{1} z+a_{0}=0$, $\left(a_{0}, a_{1}>0\right)$. We obtain

$$
\begin{aligned}
15^{\circ}<\phi_{1}<16 \frac{4}{11}^{\circ}, 45^{\circ}<\phi_{2}<49 \frac{1}{11}^{\circ}, \quad 75^{\circ}<\phi_{3}<81 \frac{9}{11}^{\circ}, \\
105^{\circ}<\phi_{4}<114 \frac{6}{11}^{\circ}, 135^{\circ}<\phi_{4}<147 \frac{3}{11}^{\circ}, 165^{\circ}<\phi_{6} \leqq 180^{\circ},
\end{aligned}
$$

with a similar distribution of 6 sectors from $180^{\circ}-360^{\circ}$. The openings of the sectors are, respectively,

$$
\frac{4}{11}^{\circ}, 4 \frac{1}{11}^{\circ}, 6 \frac{9}{11}^{\circ}, 9 \frac{6}{11}^{\circ}, 12 \frac{3}{11}^{\circ}, 15^{\circ}, 15^{\circ}, \cdots, 1 \frac{4}{11}^{\circ} \text {. }
$$

N. Obreschkoff* has given another extension of Descartes' rule. His result is as follows.

* Sur un problème de Laguerre, Comptes Rendus, vol. 177 (1923), pp. 102104. 
Let $f(z)=a_{0}+a_{1} z+\cdots+a_{n} z^{n}=0$ be an equation with real coefficients. The number $v_{a}$ of variations in the sequence of its coefficients is not only an upper bound for the number $N$ of its positive roots, but also an upper bound for the number of roots lying in the sector $|\arg z|<\pi / n$. If the number of roots lying in the sector is less than $v_{a}$, it is smaller by an even number.

I. J. Schoenberg was kind enough to let me see the manuscript of an article soon to be published in the Annals of Mathematics. In the first part of this paper, the author introduces an ingenious extension of the notion of a sequence of variations in signs to the case of complex coefficients. This is accomplished by the so-called separating double-sectors for the coefficient $a_{\nu}$. To define these, we imagine in the plane of complex numbers the coefficients $a_{v}$ marked by their corresponding points. Through the origin draw any two straight lines $l_{1}$ and $l_{2}$ that divide the plane into four consecutive sectors $A_{1}, A_{2}, A_{3}, A_{4}$, with the following property: one of two pairs of opposite sectors, for example, $A_{1}$ and $A_{3}$, shall not contain any of the points $a_{\nu}$ in their interior. If the common opening of $A_{1}$ and $A_{3}$ is $\psi,(0<\psi \leqq \pi)$, we call $A_{1}$ and $A_{3}$ together a separating double-sector (of aperture $\psi$ ) for the coefficients $a_{\nu}$. In going in order through the sequence of points $a_{0}, a_{1}, \cdots, a_{n}$, we count the number of times we have to pass from the sector $A_{2}$ to $A_{4}$ or vice-versa. This number is called $v_{a}(S)$ and is equal to the number of variations of the sequence $a_{0}, \cdots, a_{n}$ with respect to the separating double-sector $S=\left(A_{1}, A_{3}\right)$. It is evident that for all $a_{\nu}$ real, $v_{a}(S)$ (taking $\psi=\pi$ ) agrees with the ordinary number of variations. Schoenberg then derives the following theorems.

Let $f(z)=a_{0}+a_{1} z+\cdots+a_{n} z^{n}=0$, where $a_{\nu}$ is real or complex. In the plane of complex numbers, mark the points $a_{v}$ and draw $a$ separating double-sector of aperture $\psi,(0<\psi \leqq \pi)$, as can always be done. Then the number of roots lying in the sector $|\arg z|<\psi / n$ is either equal to $v_{a}(S)$, or is less by an even number. To find an upper bound for the number of roots of $f(z)=0$ lying in a sector whose bisectrix makes with the positive axis the angle $\theta$, a substitution $z=e^{i \theta} z^{\prime}$ is carried out.

For any sector with the vertex at the origin and of aperture $2 \pi /[n(n+1)]$ an upper bound of the number of roots lying in the sector can be established.

The rest of this interesting paper deals with applications of 
these ideas to equations of various types which fall outside the frame of this paper.

6. Absolute Value, Argument, Real Part, Pure Imaginary Part of Complex Roots.

Given

$$
f(z)=a_{0} z^{n}+a_{1} z^{n-1}+\cdots+a_{n}=0,
$$

where $z=\alpha+i \beta=r e^{i \phi}$ is a root. It is well known that, if $a_{\nu}$ are integers, then $r, \cos \phi, \sin \phi, \alpha$, and $\beta$ all are algebraic numbers, and satisfy equations with integral coefficients which may be derived from $f(z)=0$ by rational operations. It would be of some interest to have a systematic examination of these equations and their use for the determination of complex roots. At present the information is badly scattered in the literature.*

Difficulties arise in all cases: (1) because the equation for any of $r, \cos \phi, \sin \phi, \alpha, \beta$ is of high degree, usually $n(n-1)$, with reductions in some cases for real rational $a_{\nu}$ : for example, for $a_{0} z^{2}+a_{1} z+a_{2}=0, z=\alpha+i \beta, \beta \neq 0, \beta$ satisfies a second degree equation, but $\alpha$ satisfies a linear equation, with integral coefficients; (2) because the equations necessarily have a large number of extraneous roots, which are sometimes not readily distinguishable from the roots required. Some aspects of a systematic examination and classification of methods of solution along these lines are contained in two master's theses. $\dagger$

It is interesting to note that the equations for $r$ and for $e^{i \phi}$ are related in structure (for $a_{\nu}$ real). Let $z=r e^{i \theta}=r s$ be a complex root of $f(z)=0$.

Then $r / s$ is also a root, and hence

$$
\begin{aligned}
a_{0} r^{n} s^{n}+a_{1} r^{n-1} s^{n-1}+\cdots+a_{n-1} r s+a_{n} & =0 \\
a_{0} r^{n}+a_{1} r^{n-1} s+\cdots+a_{n-1} r s^{n-1}+a_{n} s^{n} & =0 .
\end{aligned}
$$

Eliminating, we have as equations for $r$ and $s$, respectively,

* References are too numerous to mention. We refer to just one interesting paper by Hayashi, Tôhoku Mathematical Journal, vol. 3 (1913), pp. 110-115, for the derivation of the equation for the absolute values. The degree of the resulting equation is incorrectly given $[n$, instead of $n(n-1)]$.

$\dagger$ R. C. Huffer, University of Illinois, 1920, and A. W. Randall, University of Colorado, 1929. 


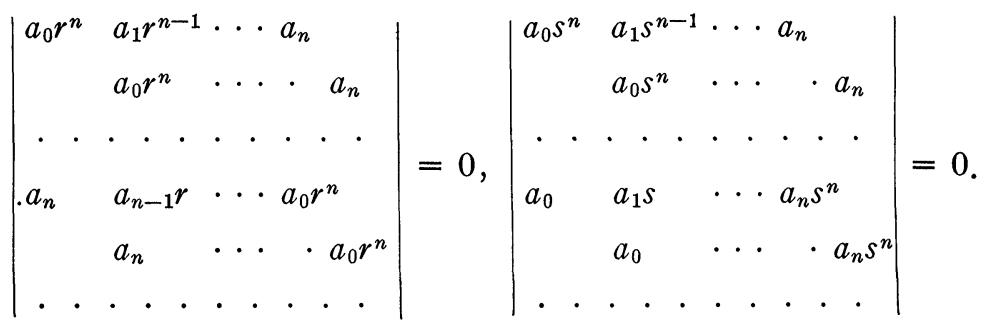

In connection with the problem of numerical solution of algebraic equations, I should like to mention a doctoral thesis* by A. J. Lewis on the solution of algebraic equations by infinite series, since it has not yet been published. As in practically all theoretical work on solutions by series, the background is given by Lagrange's expansion. The method was elaborated by Nekrassoff (1887), McClintock (1894), Lambert (1908), and others. However, the whole work is very fragmentary in character; and Lambert's work, in particular, is open to criticism. Lewis has given a more detailed treatment of the expansions than was contained in the literature, has corrected Lambert's errors, and has made a step in advance in the investigation of the convergence conditions. Trinomial and quadrinomial equations are discussed in detail, and the treatment for equations with more than four terms is indicated. The formulas are very complex, but this is due to the intrinsic difficulties of the problem.

7. On a Graphical Method for the Separation and Computation of Complex Roots. The next article I shall take the liberty of reporting more in detail, since it is published in a journal not easily available to mathematicians. $\dagger$ In this paper, three methods of graphical representation are compared. $\ddagger$

(1) The representation familiar from conformal mapping, or from proofs of the Fundamental Theorem of Algebra: $f(z)$ $=f(x+i y)=u(x, y)+i v(x, y)$. The intersections of the two curves $u(x, y)=0, v(x, y)=0$ in the $x y$ plane give the roots $x+i y$ of $f(z)=0$. In order to find the number of roots of absolute value $\rho$, where $\rho$ is arbitrarily assigned, it is only necessary to

* A. J. Lewis, Thesis, University of Colorado, 1932.

$\dagger$ Kempner, On the separation and computation of complex roots of algebraic equations, The University of Colorado Studies, vol. 16 (1928), pp. 75-87.

$\ddagger$ For the careful computation of all graphs of this paper, I am under great obligation to my colleague Professor C. A. Hutchinson. 
find the number of points of intersection of the $u$ curve and the $v$ curve inside a circle about the origin of radius $\rho$.

It is possible, by means of Kronecker's characteristics, or by equivalent methods, to determine from the figure the number of roots of $f(z)=0$ inside any closed contour by considering nothing but the points on the contour where the curves $u=0$ and $v=0$ enter and leave the region. We shall use this idea later.

(2) Let

$$
f(z)=c_{n} z^{n}+c_{n-1} z^{n-1}+\cdots+c_{0}=0,
$$

where $c_{k}=a_{k}+i b_{k}=r_{k}\left(\cos \gamma_{k}+i \sin \gamma_{k}\right)=r_{k} e^{i \gamma_{k}}$, and $z=x+i y$ $=\rho(\cos \phi+i \sin \phi)=\rho e^{i \phi}$.

We plot

$$
u=\sum_{k=1}^{n} r_{k} \rho^{k} \cos \left(k \phi+\gamma_{k}\right), \quad v=\sum_{k=1}^{n} r_{k} \rho^{k} \sin \left(k \phi+\gamma_{k}\right)
$$

in the following manner. For $\rho$, the absolute value of $z$, we select an assigned value which is held fixed. Then $u$ and $v$ become functions of $\phi$ alone, say $u=\psi_{1}(\phi), v=\psi_{2}(\phi)$. Thus $u$ and $v$ may be considered as coordinates of a curve given in parametric form, with $\phi$ as the parameter. We plot the curve in a $u v$ system of coordinates, choosing the horizontal axis as the $u$ axis and the vertical axis as the $v$ axis.

(3) We now plot the two curves $u=\psi_{1}(\phi), v=\psi_{2}(\phi)$ separately, but both in the same system of coordinates. For both curves, we take the horizontal axis as the $\phi$ axis, the vertical axis as the $u$ axis for the first curve, and as $v$ axis for the second curve.

Discussion of (2). To a circle in the complex $z$ plane about the origin, of given radius $\rho$, corresponds in the $u v$ plane, which we may also interpret as the $(u+i v)$ plane, a certain closed curve. If $N$ is the number of roots of $\psi(z)=0$ in the circle, and if we assume that no roots lie on the circumference, each root being counted with its proper multiplicity, then $2 \pi N$ represents, as is known from the theory of analytic functions of a complex variable, the change of amplitude when the curve is once completely traced in the $(u+i v)$ plane.

Simple considerations of a type familiar from the theory of characteristics will permit us to read off this number $N$ from our representation (2). To find the number of roots in a circle of radius $\rho$ about the origin in the $z$ plane, we consider the corresponding closed curve (2). Trace the curve (in either the posi- 
tive or negative sense), starting from an arbitrarily chosen point, and mark as points $\beta$ all points where the curve crosses the $u$ axis $(v=0)$, and as points $\alpha$ all points where it crosses the $v$ axis $(u=0)$. Now we determine the change in the argument between two consecutive points of intersection with the $u$ axis, as we move along the curve. We call this argument $\psi$, since it is not necessarily the same as the parameter $\phi$, the argument of the construction (3). The simplest case occurs when our points $\alpha$ and $\beta$ form an alternating sequence, $\alpha \beta \alpha \beta \ldots \alpha \beta$. To a change from any $\alpha$ to the next $\alpha$ corresponds each time a change of $\pi$ in $\psi$. All these changes are in the same sense; that is, $\psi$ is either each time increased by $\pi$ or each time decreased by $\pi$. Hence $\psi$ increases or decreases by $m \pi$, where $m$ is the number of $\beta$, and there are therefore $m / 2$ roots in the interior of the circle of radius $\rho$ about the origin in the $(x+i y)$ plane. But the number $m=2 N$ is correctly expressed by the following theorem for the special case $\epsilon_{1}=\epsilon_{2}=\cdots=\epsilon_{\nu}=1, \nu=m$.

Theorem 10. Denote by $\beta_{1}, \beta_{2}, \cdots$ the points of intersection of curve (2) with the $u$ axis, by $\alpha_{1}, \alpha_{2}, \cdots$ the points of intersection with the $v$ axis, and consider the chain of the points $\alpha$ and $\beta$ cyclically closed. If then, starting from any $\alpha$, the number of $\beta$ points between two consecutive $\alpha$ points is, respectively, $\epsilon_{1}, \epsilon_{2}, \cdots, \epsilon_{k}$, then the number of roots $z$ of $f(z)=0$ for which $|z|<\rho$ is given by the formula

$$
2 N=\left|\sum_{k=1}^{\nu}(-1)^{\left(\epsilon_{1}+1\right)+\left(\epsilon_{2}+1\right)+\cdots+\left(\epsilon_{k}+1\right)}\right| .
$$

I omit the proof that this formula, derived for the special case of alternating $\alpha$ and $\beta$, is true for any sequence of the $\alpha$ and $\beta$. These considerations lead to the following rule.

RuLE. Write down, in proper order, the numbers which represent the number of $\beta$ in the successive $\alpha$-intervals. Under the first of these numbers write +1 , if it is odd, or -1 , if it is even. Write then under each odd number that one of the two numbers, +1 or -1 , which is already written under the last preceding number; but under each even number write +1 when -1 is written under the last preceding number, and write -1 when +1 was written under the preceding number. The sum of all of these numbers $+1,-1$ is necessarily even, and the absolute value of the sum is $2 \mathrm{~N}$. 
Discussion of (3). From the constructions (2) and (3), we find the following relation between them.

It is clear that to every curve (3) will correspond uniquely a curve (2), since for every value of $\phi$ both $u$ and $v$ are uniquely determined. On the other hand, from a curve (2) a curve (3) is not uniquely determined: according to the manner of parametric representation of $u$ and $v$ by means of $\phi$, an infinite number of curves (3) may be obtained. All curves (3), however, which correspond to the same curve (2), are related to one another in a simple fashion.

The theorem is applied, without modification, to (3). In our curves (1), drawn for a given value $\rho$, and with a common $\phi$ axis, the intersections of the $u$ curve with the $\phi$ axis and the intersections of the $v$ curve with the $\phi$ axis correspond in a definite manner to the intersections of the $u v$ curve in (2) with the $u$ axis and the $v$ axis. To make this clear, it is only necessary to consider carefully a special case. Consider the $u v$ curve treated in Fig. 12. ${ }^{*}$ To this figure corresponds (schematically) Fig. $2_{3}$ as is immediately checked. In comparing these two figures we have only to keep in mind that every time the curve (2) crosses the $u$ axis, we have $v=0$, so that in (3) the $v$ curve crosses the $\phi$ axis, and that every time the curve (2) crosses the $v$ axis, we have $u=0$, so that in (3), the $u$ curve crosses the $\phi$ axis, and finally, that the combined sequence of the points of intersection of the $u$ curve and the $v$ curve with the $\phi$ axis follow in the same order as the $\alpha \beta$ sequence in (2).

It follows that we get exactly the same $\alpha \beta$ sequence, whether we read it off from (2) or from the sequence obtained by marking in (3) as $\alpha$ all points of intersection of the $u$ curve with the $\phi$ axis, and as $\beta$ all points of intersection of the $v$ curve with the $\phi$ axis. We have, therefore, the following theorem.

Theorem 11. Plot, as explained under (3), the curves

$$
u=\sum_{k=0}^{n} r_{k} \rho^{k} \cos \left(k \phi+\gamma_{k}\right), \quad v=\sum_{k=0}^{n} r_{k} \rho^{k} \sin \left(k \phi+\gamma_{k}\right),
$$

for a given $\rho$. Name the points of intersection of the u-curve with the $\phi$ axis, $\alpha_{1}, \alpha_{2}, \cdots$, and name the points of intersection of the $v$ curve with the $\phi$ axis, $\beta_{1}, \beta_{2}, \cdots$. Consider the combined se-

* The index 2 in $1_{2}$ indicates that the figure refers to construction (2), etc. 
quence of the $\alpha$ and $\beta$ points in their natural order of magnitude. Assume the sequence closed cyclically, and let $\epsilon_{1}, \epsilon_{2}, \cdots, \epsilon_{\nu}$, respectively, be the number of $\beta$ points between two consecutive $\alpha$ points; then the number $N$ of roots of $f(z)=0$ for which $|z|<\rho$ is given by the formula

$$
2 N=\left|\sum_{k=1}^{\nu}(-1)^{\left(\epsilon_{1}+1\right)+\left(\epsilon_{2}+1\right)+\cdots+\left(\epsilon_{k}+1\right)}\right| .
$$

This number may be evaluated by the rule stated under Theorem 10.

Example. The following example is only schematic, and shows how the rule and the theorem are applied to more complicated cases.

Figures $1_{2}$ and $2_{3}$ are corresponding figures. From either of them we read off the $\alpha \beta$ sequence, and apply the rule or the theorem.

$$
\epsilon_{k}=\begin{array}{crrrrrrrr}
\beta \alpha \alpha & \beta & \alpha \alpha \alpha & \beta & \alpha & \beta & \beta & \beta & \beta \\
0 & 1 & 0 & 0 & 2 & 1 & 1 & 3 \\
-1 & -1 & +1-1 & +1 & +1 & +1 & +1
\end{array}
$$

Using the rule, we find $2 N=2, N=1$.

Using the theorem, we find

$$
\begin{aligned}
& \begin{array}{lllllllll}
\epsilon_{k}+1= & 1 & 2 & 1 & 1 & 3 & 2 & 2 & 4
\end{array} \\
& 2 N=(-1)^{1}+(-1)^{3}+(-1)^{4}+(-1)^{5}+(-1)^{8}+(-1)^{10}+(-1)^{12} \\
& +(-1)^{16}=2, \quad N=1 \text {. }
\end{aligned}
$$

If we had started with $\beta$ intervals and had considered the number of $\alpha$ in each $\beta$ interval, we should have found

$$
\begin{aligned}
& \begin{array}{lllllllll}
\beta \alpha \alpha \alpha \alpha \alpha & \beta & \alpha & \alpha & \alpha & \beta & \alpha & \beta
\end{array}
\end{aligned}
$$

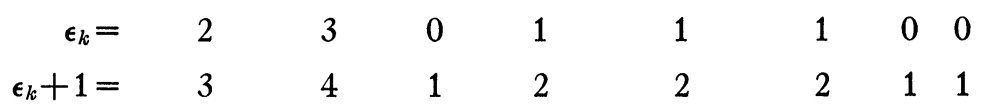

$$
\begin{aligned}
& 2 N=(-1)^{3}+(-1)^{7}+(-1)^{8}+(-1)^{10}+(-1)^{12}+(-1)^{14}+(-1)^{15} \\
& +(-1)^{16}=2, \quad N=1 \text {. }
\end{aligned}
$$

Application of Theorem 11. We make use of the theorem to isolate the complex roots of an equation in concentric rings $\left(\rho_{1}<\rho<\rho_{2}\right)$ around the origin as center. The procedure is as follows. For two given positive values of $\rho, \rho_{2}>\rho_{1}$, we assume 


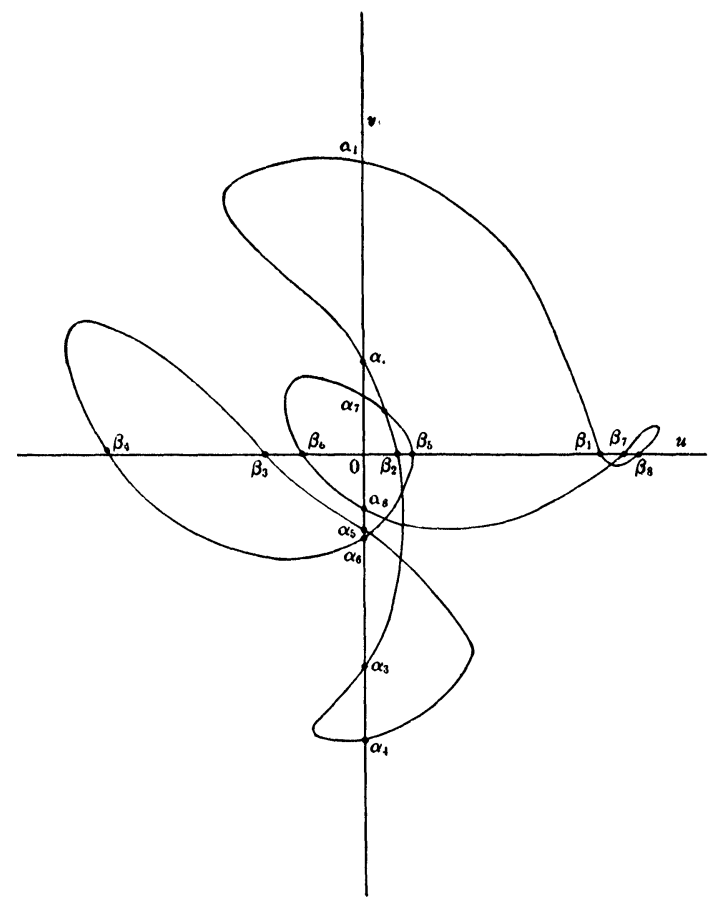

FIG. $1_{3}$

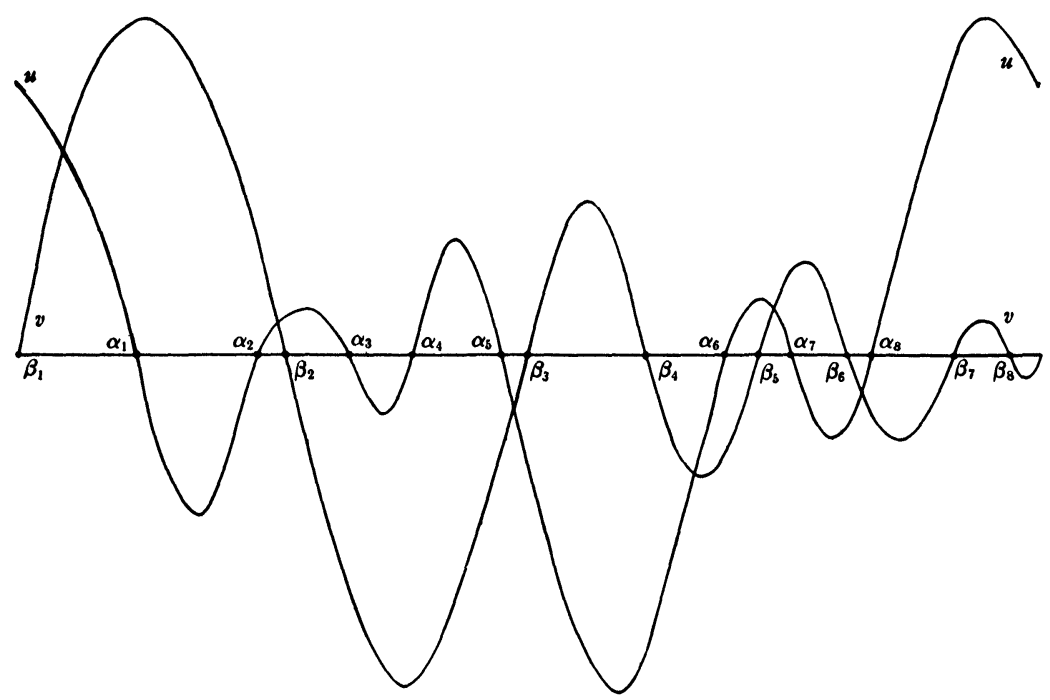

FIG. $2_{3}$ 
that the curves $u(\phi)$ and $v(\phi)$ are drawn in our system (3). By Theorem 10 we read off the numbers $N\left(\rho_{1}\right), N\left(\rho_{2}\right)$ of roots of absolute value smaller than $\rho_{1}$ and $\rho_{2}$, respectively. Then $N\left(\rho_{2}\right)$ $-N\left(\rho_{1}\right)$ gives the exact number of roots in the ring-region $\rho_{2}>\rho>\rho_{1}$. Here we assume, since this case is easily disposed of, that no root lies on the circumference of either of the circles $\rho_{1}$ or $\rho_{2}$.

In case we have the same sequence of $\alpha$ and $\beta$ for $\rho_{1}$ as we have for $\rho_{2}$, there are obviously no roots in the ring-shaped region $\rho_{1}<|z|<\rho_{2}$.

Since there exist well known simple upper and lower bounds for the absolute value of the roots of a given equation, we may start out with two such values for $\rho$, and, by appropriate choice of new values of $\rho$, we can separate our roots according to their absolute value.

It is a simple matter to plot curves of the kind used here, particularly because we are really interested only in the se-

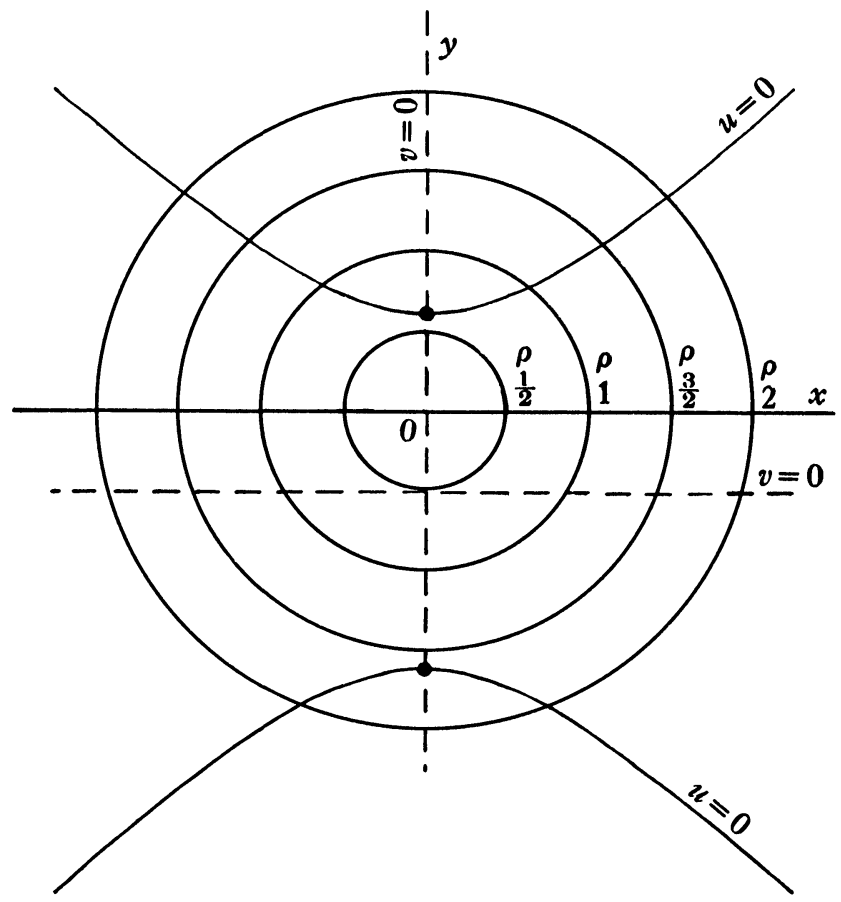

FIG. $3_{1}$ 
quence of the points of intersection of the $u$ curve and the $v$ curve with the $\phi$ axis. The chief advantage of the present method may lie in the fact that instruments exist with which curves of type (3) can be drawn. Such an instrument is the Michelson-Stratton harmonic analyzer, for which an accuracy of about 1 per cent to 2 per cent is claimed.

Example. Consider the equation

$z^{2}+i z+1=0, u=\rho^{2} \cos 2 \phi-\rho \sin \phi+1, v=\rho^{2} \sin 2 \phi+\rho \cos \phi$.

Figure $3_{1}$ gives the classical construction, which may be used for the verification of our results. Figure $4_{2}$ gives, for $\rho=1 / 2$, the parametric representation of $u=u(\phi), v=v(\phi)$ in a $u v$-system of coordinates, while Fig. $5_{3}$ gives $u$ and $v$ both as functions of $\phi$. Figures $6_{2}$ and $7_{3}$ are the corresponding curves for $\rho=1$; Figs. $8_{2}$ and $9_{3}$ are the curves for $\rho=3 / 2$; and finally Figs. $10_{2}$ and $11_{3}$ are the curves for $\rho=2$.

For $\rho=1 / 2$, from Fig. $5_{3}$, we have

$$
\begin{gathered}
\beta \beta \\
\epsilon_{k}=00, \quad 2 N=(-1)^{1}+(-2)^{2}=0, \quad N=0 .
\end{gathered}
$$

This is obvious, since the $u$ curve does not intersect the $\phi$ axis at all. For $\rho=1$, from $7_{3}$, we find

$$
\epsilon_{k}=\begin{array}{ccc}
\alpha \beta \alpha \beta \beta \beta & 1 & 3
\end{array}, \quad 2 N=(-1)^{2}+(-1)^{4}=2, \quad N=1 .
$$

For $\rho=3 / 2$, from $9_{3}$, we get

$$
\alpha \beta \alpha \beta \beta \beta, \quad N=1, \text { as for } \rho=1 .
$$

For $\rho=2$, from $11_{3}$, we obtain

$$
\begin{aligned}
& \alpha \beta \alpha \beta \alpha \beta \alpha \beta \\
& \epsilon_{k}=\begin{array}{lllll}
1 & 1 & 1 & 1
\end{array} \\
& 2 N=(-1)^{2}+(-1)^{4}+(-1)^{6}+(-1)^{8}=4, \quad N=2 \text {. }
\end{aligned}
$$

Therefore, the equation $z^{2}+i z+1=0$ has one root in the ringregion $1 / 2<\rho<1$ and one in the region $3 / 2<\rho<2$. This agrees with Fig. $3_{1}$. The absolute values of the two roots are $5^{1 / 2} / 2$ $-1 / 2=0.6+$ and $5^{1 / 2} / 2+1 / 2=1.6+$, respectively.

It will be recognized immediately that the graphs shown in 
I935.]

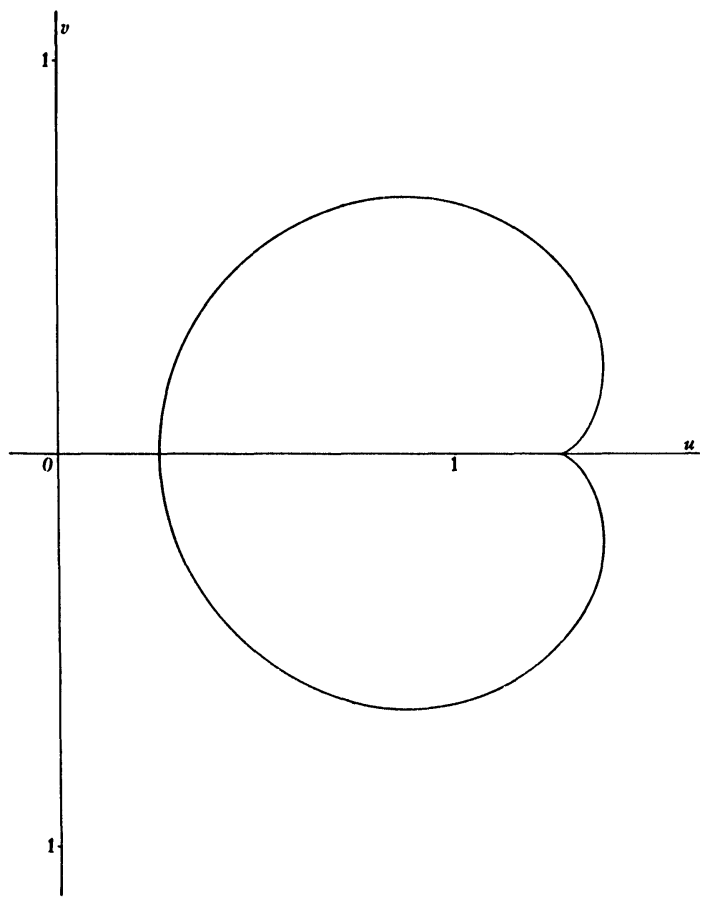

FIG. $4_{2}$

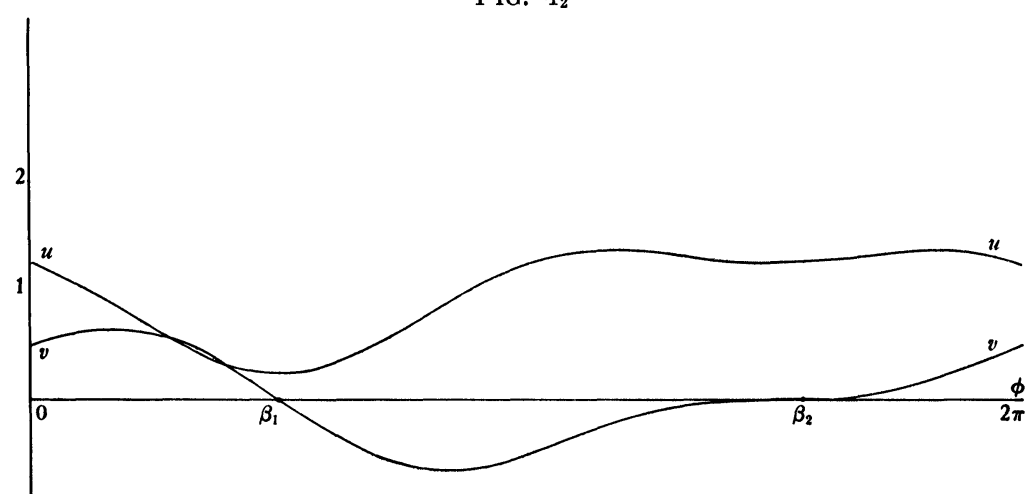

FIG. $5_{3}$ 
Figs. $5_{3}, 7_{3}, 9_{3}, 11_{3}$ also give information concerning the $\arg u$ ments of the complex roots.

8. Separation of Roots in Sectors. Without insisting at the present time, I shall only indicate how these same ideas can be utilized to separate roots in sectors from the origin by interchanging the role of $r$ and $\phi$ in the former process.

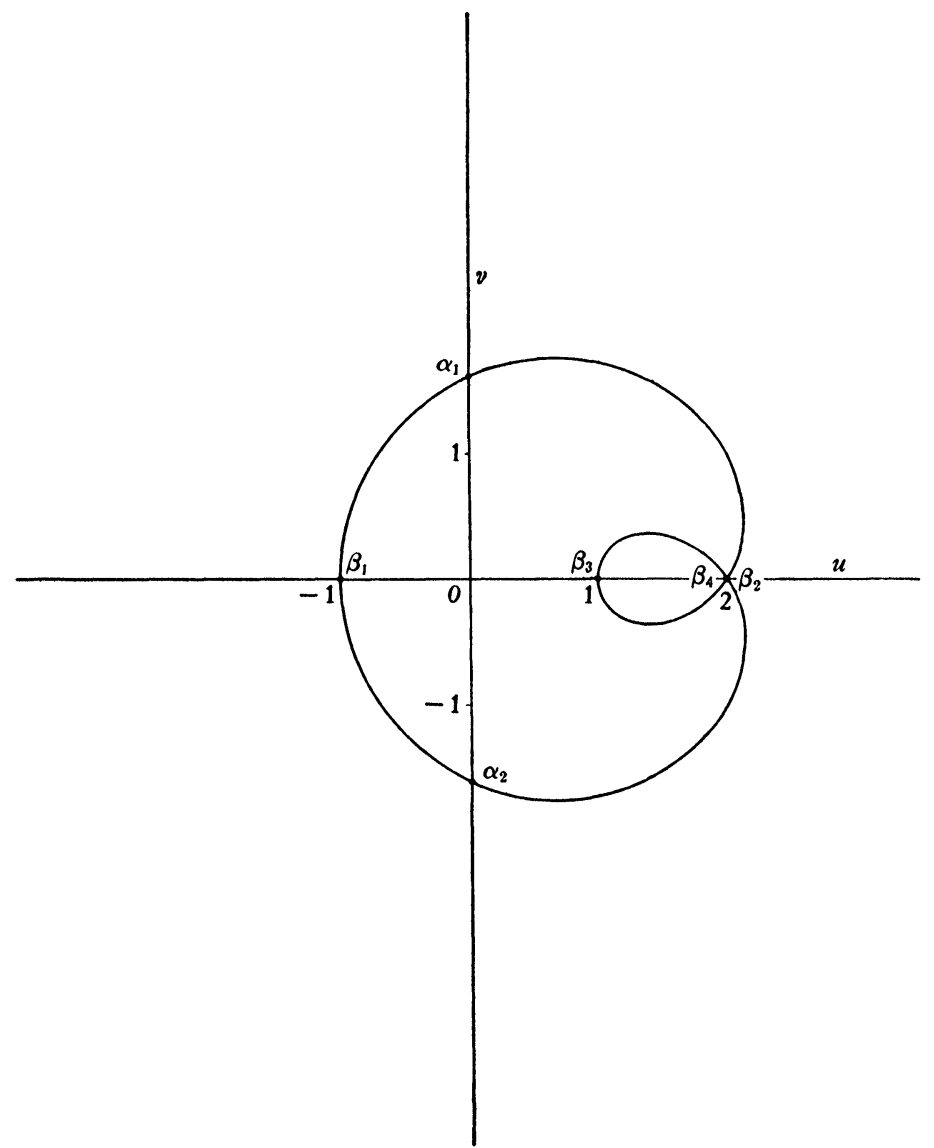

FIG. $6_{2}$

Given the equation $f(z)=a_{0} z^{n}+\cdots+a_{n}=0$, where $a_{v}$ is real or complex, and again $z=\rho e^{i \phi}=\rho(\cos \phi+i \sin \phi)$. Now, for a given $\phi$, we have $u=u(\rho), v=v(\rho)$. Plot $u(\rho)$ and $v(\rho)$ in the same system of coordinates, with $\rho$ as horizontal axis, and with $u$ 
and $v$ as vertical axes. We know how to find upper and lower bounds $\rho_{1}$ and $\rho_{2}$ for the absolute value of the roots. The value of $\rho$ (always $>0$ ) is to be restricted to the interval between these upper and lower bounds. If the sequence of $\alpha$ and $\beta$ is the same in the interval $\left(\rho_{1}, \rho_{2}\right)$ for $\phi=\phi_{1}$ and for $\phi=\phi_{2}$, there are no roots of $f(z)=0$ in the sector $\phi_{1} \leqq \phi \leqq \phi_{2}$. If the sequence of $\alpha$ and $\beta$ is not the same for $\phi=\phi_{1}$ and $\phi=\phi_{2}$, there are roots in the sector and the exact number may be read off by the same kind

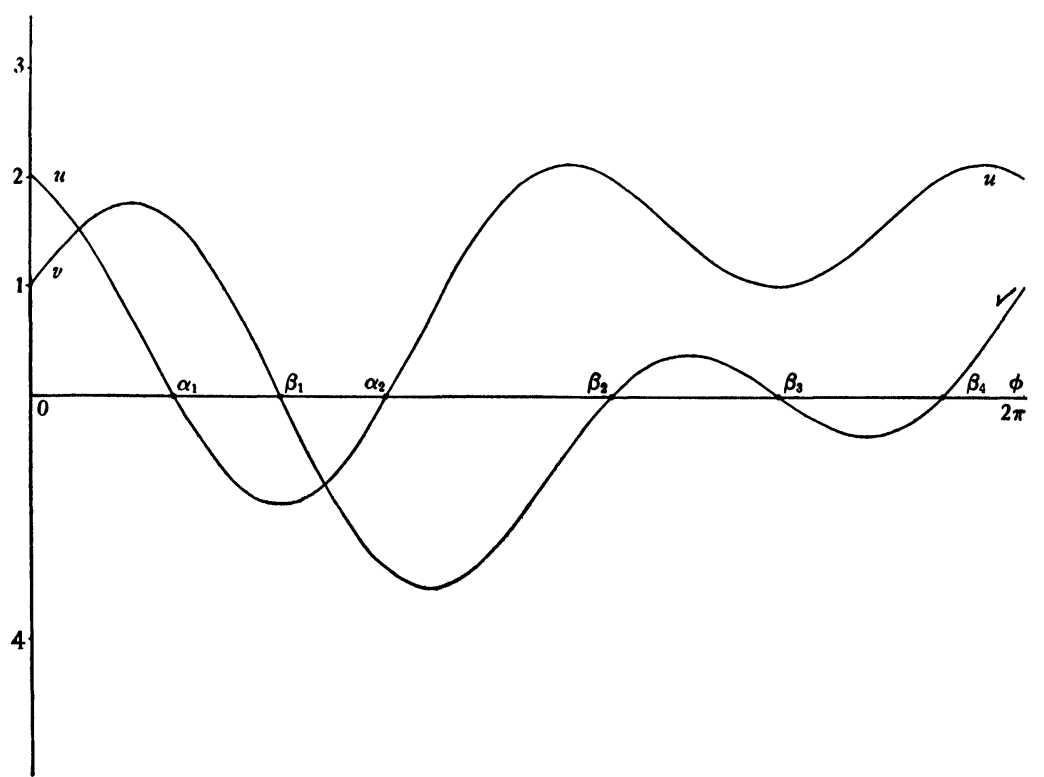

FIG. 7 s

of theorem or rule as that which was derived above. The details of this are reserved for another occasion. At present, we restrict ourselves to the verification that $z^{3}-2 z-2=0$ has a root in the sector $3 \pi / 4 \leqq \phi \leqq \pi$, with $1<\rho<3 / 2$ :

$$
z^{3}-2 z-2=0 \text {. }
$$

$u=\rho^{3} \cos 3 \phi-2 \rho \cos \phi-2, \quad v=\rho^{3} \sin 3 \phi-2 \rho \sin \phi$.

$\phi=3 \pi / 4: \quad u=\frac{1}{2}(2)^{1 / 2} \rho^{3}+(2)^{1 / 2} \rho-2, \quad v=\frac{1}{2}(2)^{1 / 2} \rho^{3}-(2)^{1 / 2} \rho ;$

$\phi=\pi: \quad u=-\rho^{3}+2 \rho-2, \quad v=\rho^{3} \sin \pi-2 \rho \sin \pi$. 
Since for $\phi=\pi, v$ reduces to 0 identically, we select $\phi=\pi-\epsilon$, where $\epsilon$ denotes some small positive angle. Then, from the relation $\lim _{\epsilon \rightarrow 0}[(\sin \epsilon) / \epsilon]=1$, we find $v=3 \rho^{3} \epsilon-2 \rho \epsilon$, approximately.

For $\phi=3 \pi / 4$, our $u$ curve, for $1<\rho<3 / 2$, lies entirely above the $\rho$ axis, and the $v$ curve crosses the $\rho$ axis once (no $\alpha$, one $\beta$ ).

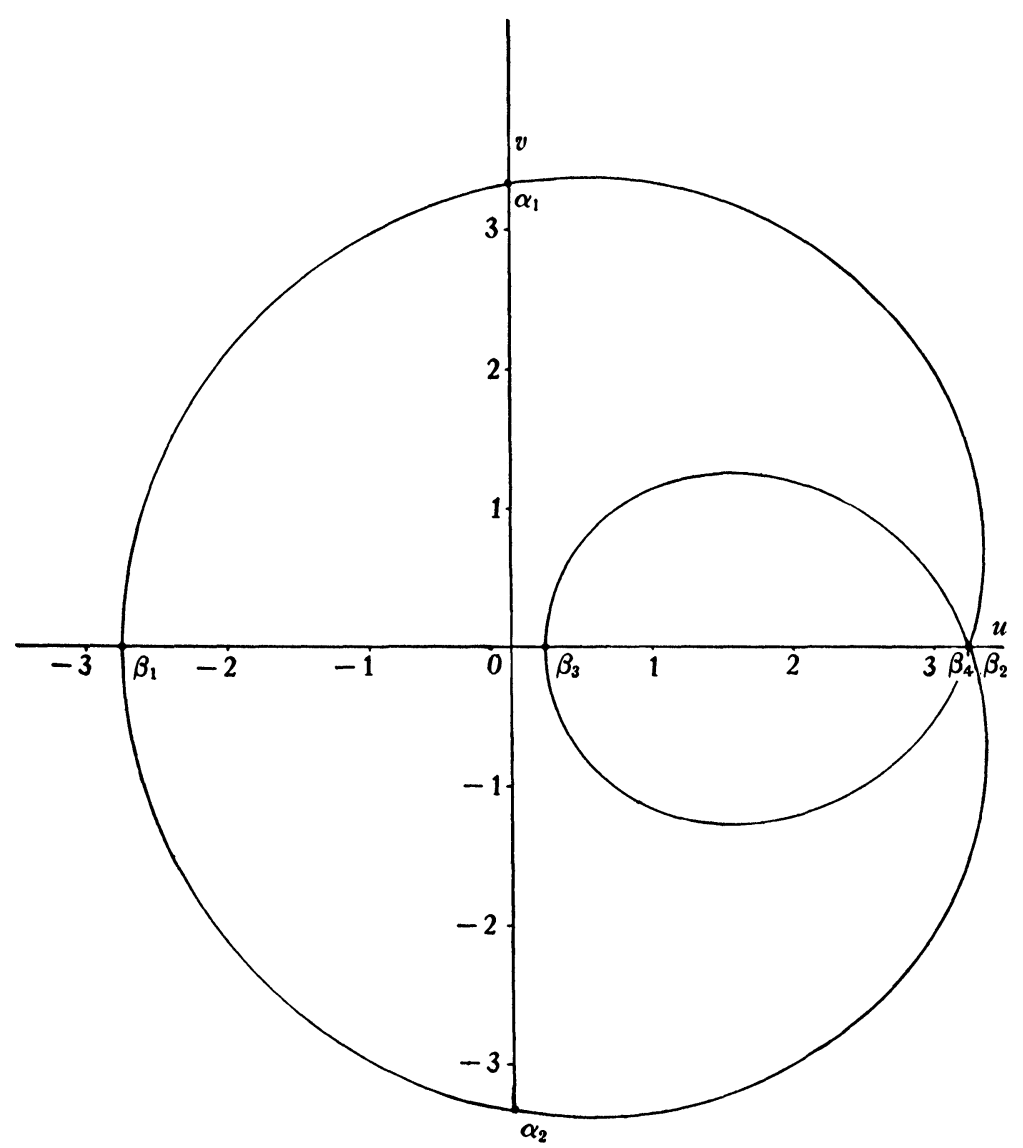

FIG. $8_{2}$

For $\phi=\pi-\epsilon$, neither the $u$ curve nor the $v$ curve cut the $\rho$ axis for $1<\rho<3 / 2$ (no $\alpha$, no $\beta$ ). There must be roots of $f(z)=0$ that lie in the sector $3 \pi / 4 \leqq \phi \leqq \pi$.

We do not have, as we did before, an instrument available for drawing the curves, but, on the other hand, they are of par- 
ticularly simple type, namely, polynomial curves. Besides we need, of course, not the curves themselves, but only their (approximate) intersections with the $\rho$ axis.

9. Polynomial Curves and Complex Roots. I shall discuss one last topic also with some degree of completeness, in order to piece together ideas which are scattered and more frequently implied than explicitly announced in the literature.

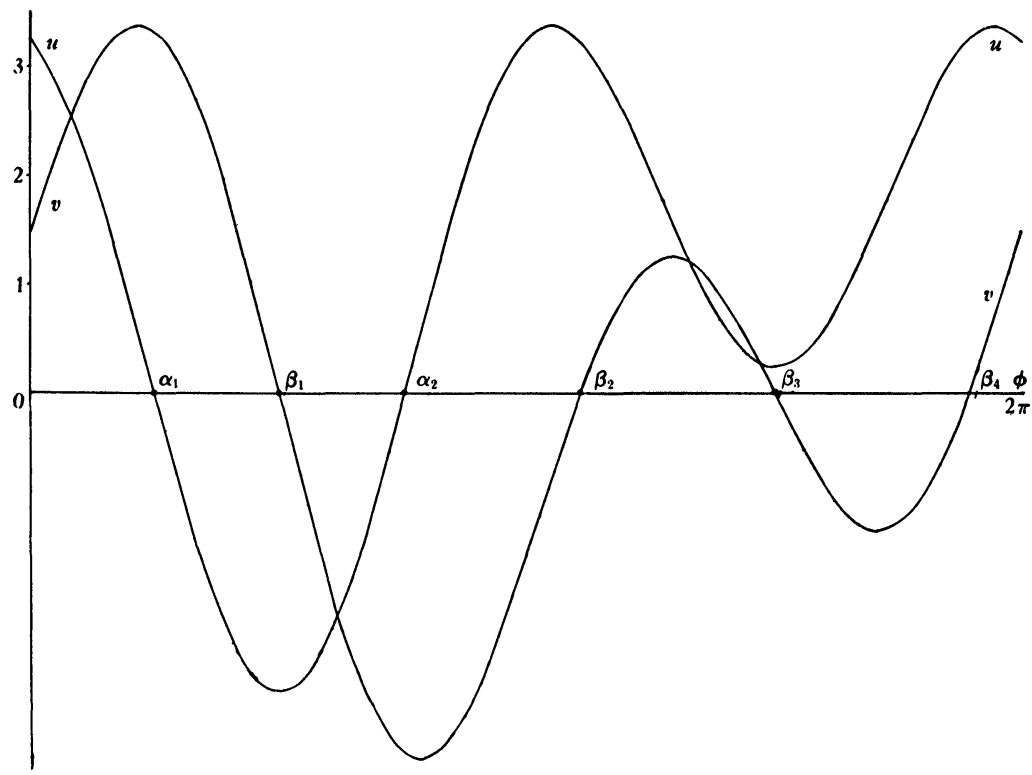

FIG. 93

The importance of polynomial curves for the real roots of algebraic equations is fundamental and obvious. But the systematic use of graphs for the purpose of locating complex roots of equations of the form $f(z)=a_{0} z^{n}+\cdots+a_{n}=0$, where $a_{v}$ is assumed, for simplicity only, to be real, is not so widely known as it should be, except for small values of $n$, such as occur in the problem of the location of complex roots of quadratic or cubic equations with real coefficients.

The character of the real graph of $f(z)$ is of great influence in some of the graphical methods for complex roots. I have classified real polynomial curves of any given degree $n$ according to 
their geometric behavior, and have given existence proofs for the various types.*

Of particular importance for our immediate purpose are certain considerations which permit us to represent in a natural

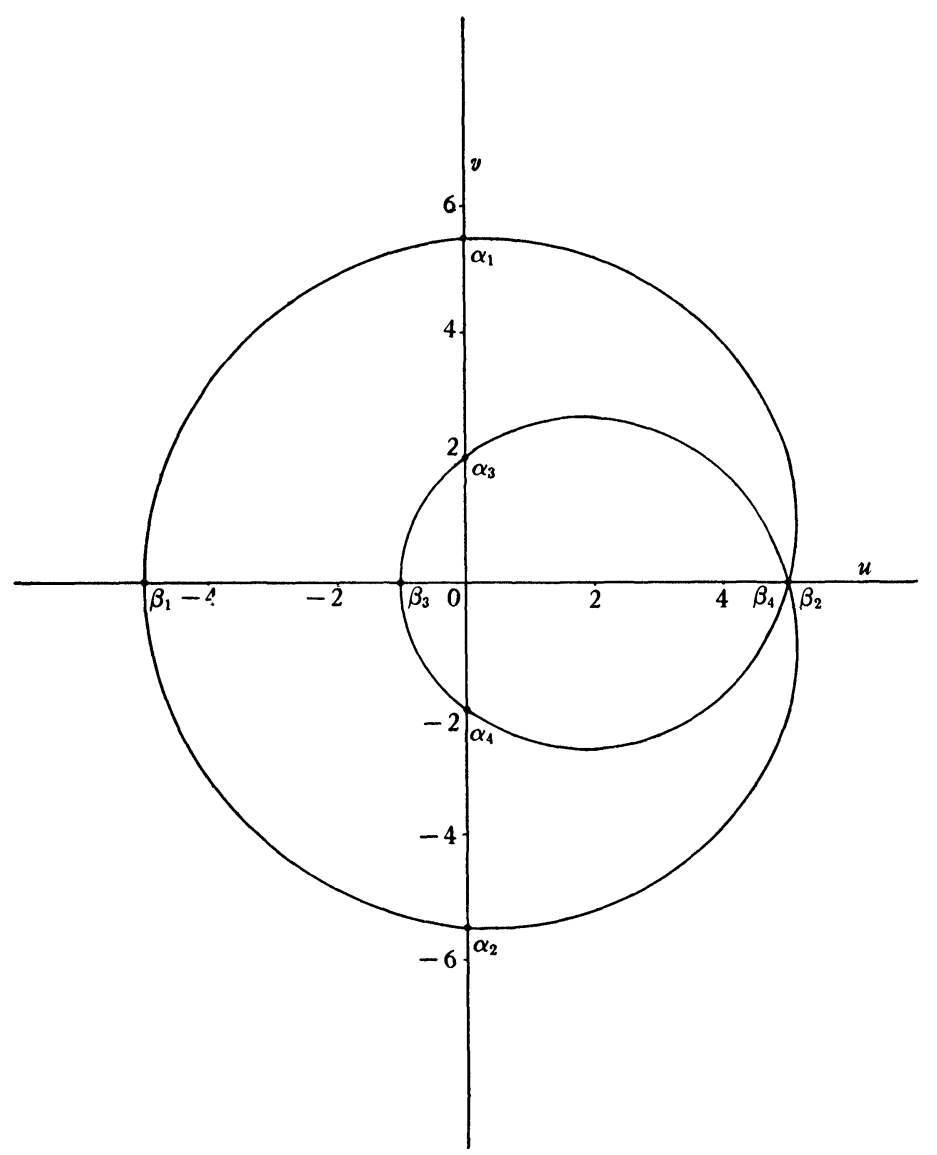

FIG. $10_{2}$

manner some parts of the complex graph, and which, although the germ of the method goes back at least to Poinsot and his

* Kempner, On the shape of polynomial curves, I. Tôhoku Mathematical Journal, vol. 37 (1933), pp. 347-362; Part II is to appear in the same journal. Compare also American Mathematical Monthly, vol. 40 (1933), pp. 469-470. 
cordes ideaux, are not given the place they deserve in modern presentations.*

The simple idea consists in an attempt to make the best of a bad situation. Since it is impossible to give in a three-dimen-

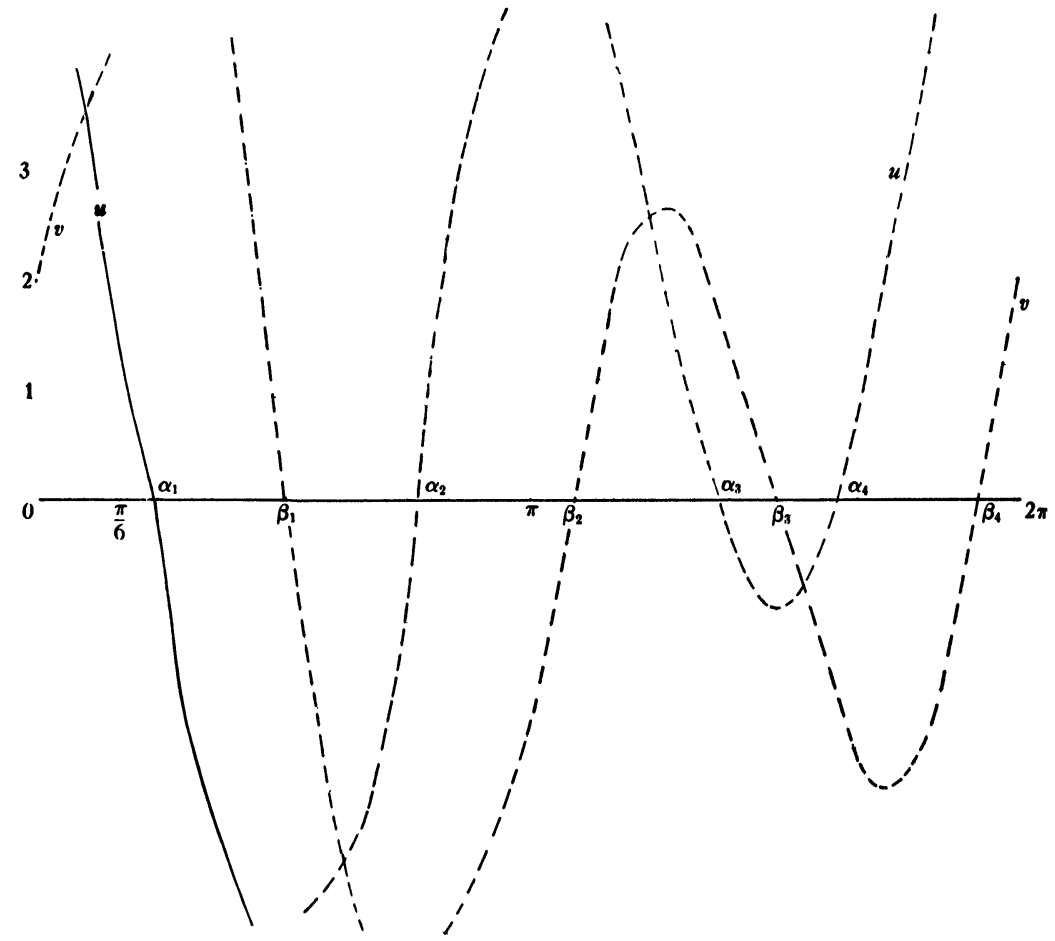

FIG. $11_{3}$

sional space a simple satisfactory representation of a function $w=f(z)$ of a complex variable, we try to save as much as we can. We may use our three dimensions as follows. Consider only real values of $f(z)$, but permit $z$ to assume all values, real and complex, which make $f(z)$ real.

Letting $f(z)=f(x+i y)=u(x, y)+i v(x, y)=u+i v$, we have,

* Phillips and Beebe, Graphical Algebra, 2nd ed. (1887) (out of print), make much use of it in an elementary study of equations of the second, third, and fourth degrees with real coefficients, but with no attempt at a systematic treatment. In particular, the curves $u(x, y)=0, v(x, y)=0$ in the $x y$ plane are not considered. The space curves in our text are to be found in Phillips and Beebe. 
for $f(z)$ real, $v(x, y)=0, f(z)=u(x, y)$. If we use a horizontal $x y$ plane for $z=x+i y$, and a vertical axis for $u(=f(z)$ real), we shall obtain a space curve in our $(x y u)$-system of coordinates. The projection of the space curve onto the $x y$ plane consists exactly of the curve $v(x, y)=0$, and the functional value is the corresponding value of $u(x, y)$.

It is evident at once that this representation is related to the theory of functions of a complex variable, since the space curve is also given as the curve of contact between the surface of absolute values $|f(z)|$ and the surface of real values $u(x, y)$ for $u(x, y)>0$, and as the curve of contact between the surfaces $-|f(z)|$ and $u(x, y)<0$. Again, the space curves may be interpreted as the intersections of the surface $u(x, y)$ with the cylindrical surface $v(x, y)=0$.

These space curves serve as a skeleton for the function. They are important for us, (a) because we can study their general character with great ease, (b) because by means of them we can always represent all roots of the equation.

(1) For a real function $f(z)=a_{0} z^{n}+\cdots+a_{n}$ with maxima and minima of the first order $\left(f^{\prime}=0, f^{\prime \prime} \neq 0\right)$ we obtain a space curve of the following type. First we have in the $x u$ plane the ordinary real graph, but in addition we have attached to each extreme a handle, symmetric to the plane of the real graph and penetrating it under a right angle. These handles always extend to infinity and their projections in the $x y$ plane are (as stated) branches of the curve $v(x, y)=0$, the properties of which are well known, sometimes under the name of Lucas' stelloids. In certain cases such a handle lies entirely in a plane, which then is for reasons of symmetry parallel to the $y u$ plane. This happens, for example, for the real quadratic function* $a z^{2}+b z$ $+c$, in agreement with the fact that $v(x, y)=0$ now consists of a line parallel to the $y$ axis, besides the $x$ axis. Similarly, for real polynomial curves with a line of symmetry, which then necessarily have a maximum or a minimum on this line, and which we assume to be an extreme of the first order, the handle attached at that extreme lies entirely in the plane perpendicular

* Leading to one of the classical constructions of complex roots of quadratic equations with real coefficients. 
to the $x u$ plane and contains the line of symmetry. For example,* consider the function $f(z)=z^{4}-2 z^{2}=\left(z-(2)^{1 / 2}\right)\left(z+(2)^{1 / 2}\right) z^{2}$. Here $v(x, y)=0$ consists of the curves $y=0, x=0, x^{2}-y^{2}-1=0$. The projection of the real part of the curve is $y=0$; the line $x=0$ contains the handle attached at the maximum; the branches of the hyperbola are the projections of the two handles attached at the minima. These last two handles therefore do not lie in planes. The distance of their points from the $x y$ plane increases monotonically as the projection moves along the hyperbola to infinity. $\dagger$

The same considerations may be applied to transcendental functions. For $\sin z$, the result is particularly simple: $v(x, y)=0$ consists in the $x y$ plane of the axis of reals (the $x$ axis) and of the equidistant lines $x= \pm(2 k+1) \pi / 2,(k=0,1,2, \cdots)$. Hence, all handles lie in planes perpendicular to the $x u$ plane and parallel to the $y$ axis. The handles are nothing but catenaries, attached at the maxima and minima, and extending upward (downward) from a maximum (minimum). Our later remarks concerning the utilization of these extended graphs for the location of complex roots of an equation $a_{0} 2^{n}+\cdots+a_{n}=0$ are immediately applicable to the location of complex roots of $\sin z=c,(c$ real, $|c|>1)$. Applied to any two real circles in a plane, the handles lead to a neat interpretation of the circular points.

For any equation of the form $a_{0} z^{n}+\cdots+a_{n}=0$ with real coefficients and with the maximum number $n-1$ of real extremes (for example, $f(z)=\left(z-\alpha_{1}\right) \cdots\left(z-\alpha_{n}\right)+\beta, \alpha_{1} \neq \alpha_{2}$ $\neq \cdots \neq \alpha_{n}$ real, $\beta$ real), the structure of the real curve and all of its handles is of such simple character that it seems a pity not to make use of it for approximating the location of complex roots. With but little practice, it is a simple matter to estimate roughly the points where this space curve intersects any plane parallel to the $x y$ plane. These points, read off in the form $x+i y$, give a first approximation to all roots of $f(z)+c=0$.

* The qualitative-not quantitative-aspects of many theorems in an interesting article by H. B. Mitchell, Transactions of this Society, vol. 19 (1918), pp. $43 \mathrm{ff}$., become intuitively evident by these geometric interpretations.

$\dagger$ See Fig. 17 for $z^{4}-2 z^{2}+1$. 
I may mention that the Fundamental Theorem of Algebra can be given a very clear interpretation by means of such space curves, and that a proof of the theorem, essentially equivalent to the proof of the existence of $n$ intersections of $u(x, y)=0$ and $v(x, y)=0$, can be constructed.

(2) It is now necessary to free ourselves from the restriction that $a_{0} z^{n}+\cdots+a_{n}$ have the maximum number of real extremes. I can only indicate the state of affairs.

To illustrate, we consider successively the functions* $f(z)$ $=z^{3}-z, z^{3}-\epsilon z, z^{3}, z^{3}+\epsilon z, z^{3}+z, 0<\epsilon<1$. As $\epsilon \rightarrow 0$, the two extremes of $z^{3}-z$ move together, coalescing for $\epsilon=0$, and leading to a point of inflection with a horizontal tangent $\left(f^{\prime}(z)=0\right.$, $\left.f^{\prime \prime}(z)=0\right)$. $\dagger$ As we pass from $z^{3}$ to $z^{3}+z$, the point of inflection is still existent, but the roots of $f^{\prime}(z)=0$ have become imaginary. (For higher degree polynomials, the points of inflection generated by the coalescence of consecutive maxima and minima may be ultimately lost.) To trace this in detail requires distinction between natural points of inflection on polynomial curves, such as must exist between any two consecutive extremes, and accessory points of inflection, due to such coalescence of consecutive maxima and minima. At a natural point of inflection, $\left|f^{\prime}(z)\right|$ is a maximum, at an accessory point, $\left|f^{\prime}(z)\right|$ is a minimum.) $\ddagger$ Schematically, $\sim, \sim$ (maximum), $\sim, \chi$ (minimum).

As $\epsilon$ goes from 1 to 0 to -1 , the handles first run together, and then completely separate from the real curve. The projections of our space curves onto the $x y$ plane are given by the corresponding equations $v(x, y)=0$.

Even if the accessory points of inflection should become lost (compare, for example, the succession of curves: $z^{4}-2 z^{2}+1$, $\left.z^{4}-\epsilon z^{2}+1, z^{4}+1, z^{4}+\epsilon z^{2}+1, z^{4}+2 z^{2}+1\right)$ these branches are still present in the space curve. They can in no way be destroyed provided $a_{0} \neq 0$, but can only be changed in shape and position and in the manner in which they are connected with each other, however the coefficients $a_{\nu}$ may vary.

* See Figs. $12-16$ for $\epsilon=1,0.1,0,-0.1,-1$, respectively.

$\dagger$ Our space curve consists of three branches, making with each other angles of $\pi / 3$ at their point of intersection, $z=0$, as is known from the theory of conformal mapping.

$\ddagger$ Kempner, Tôhoku Mathematical Journal, loc. cit. (On the shape of polynomial curves.) 


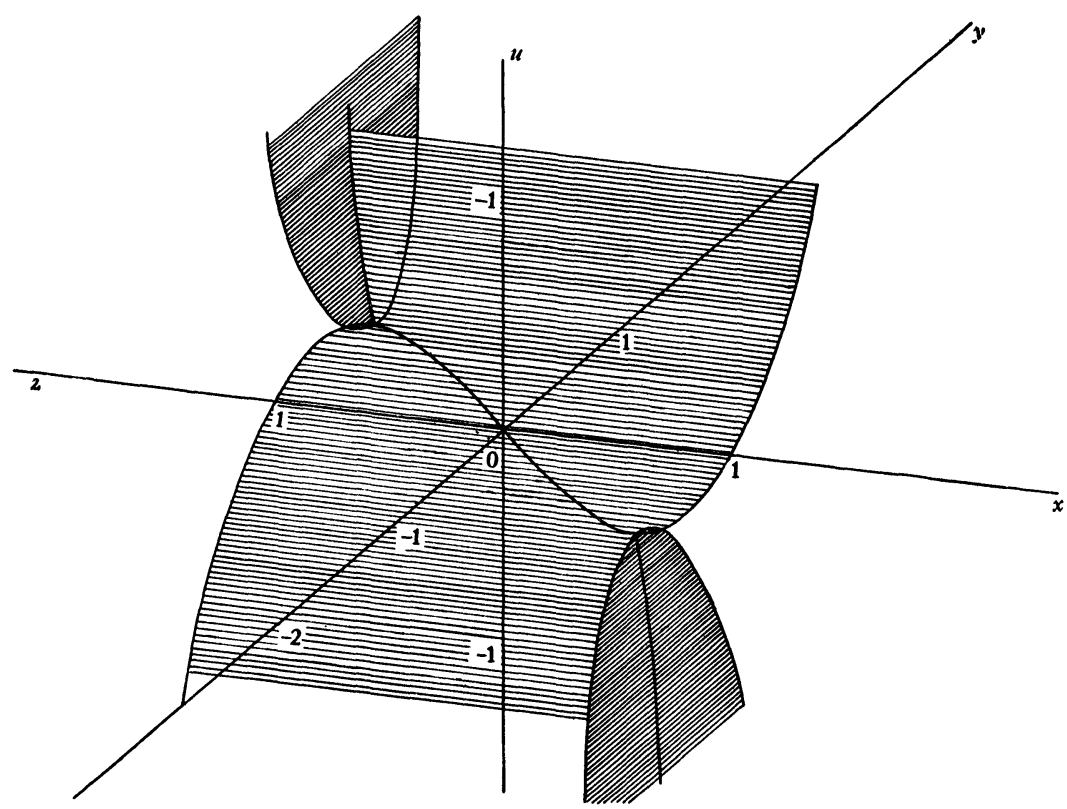

FIG. 12

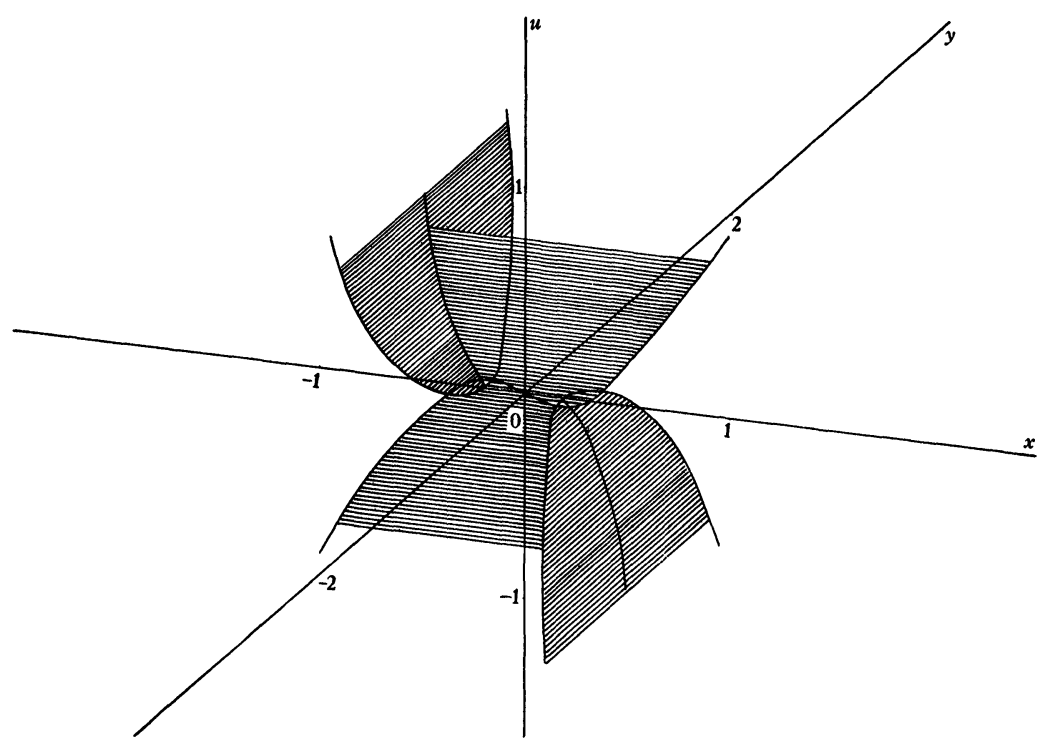

FIG. 13 


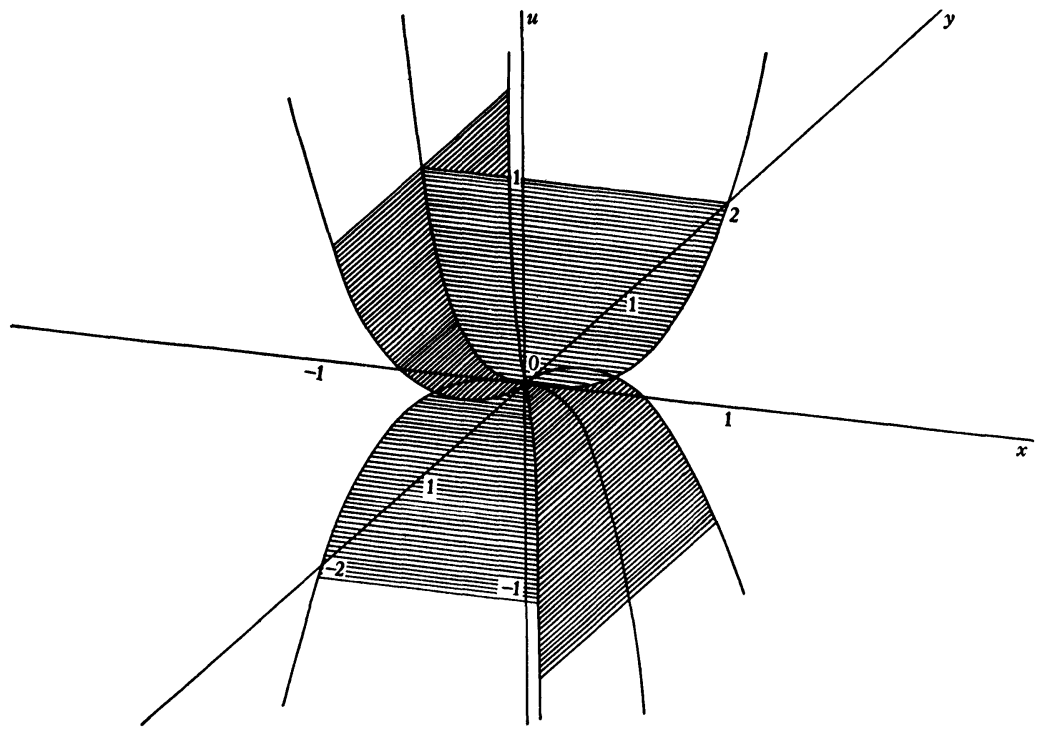

FIG. 14

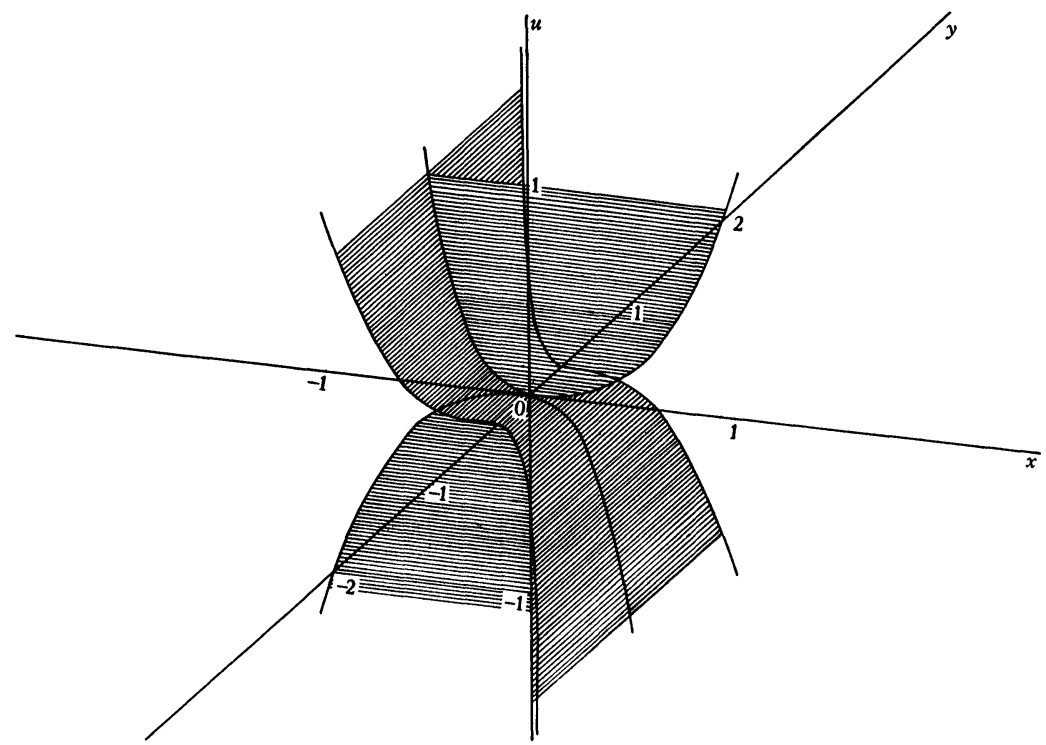

FIG. 15 
1935.]

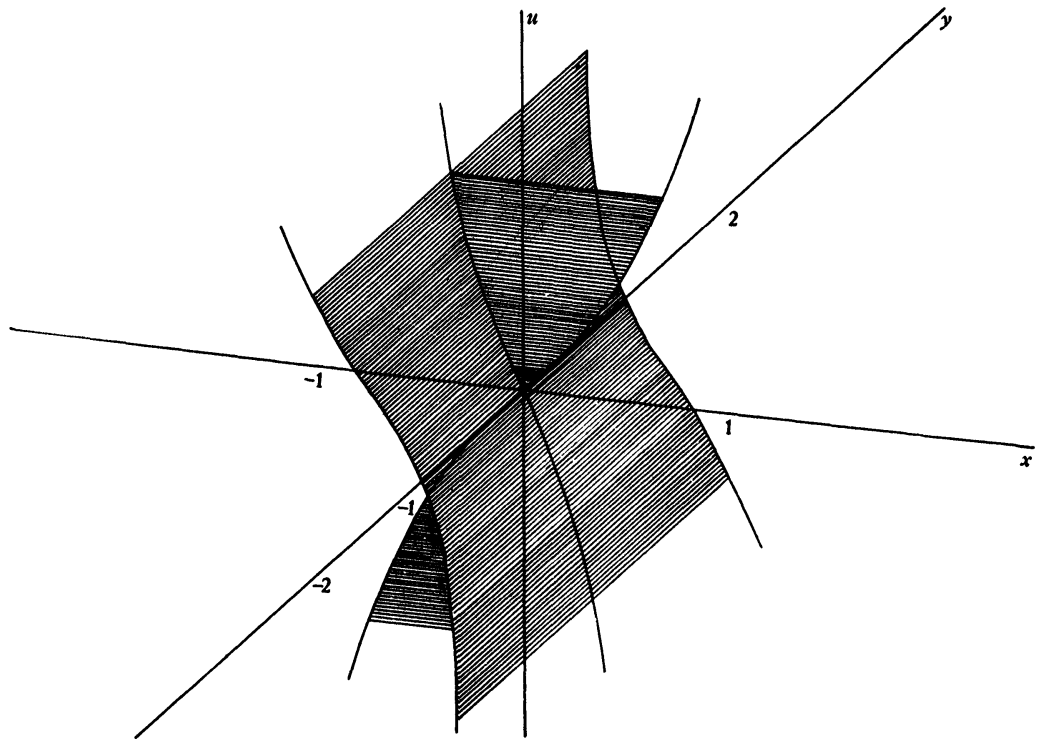

FIG. 16

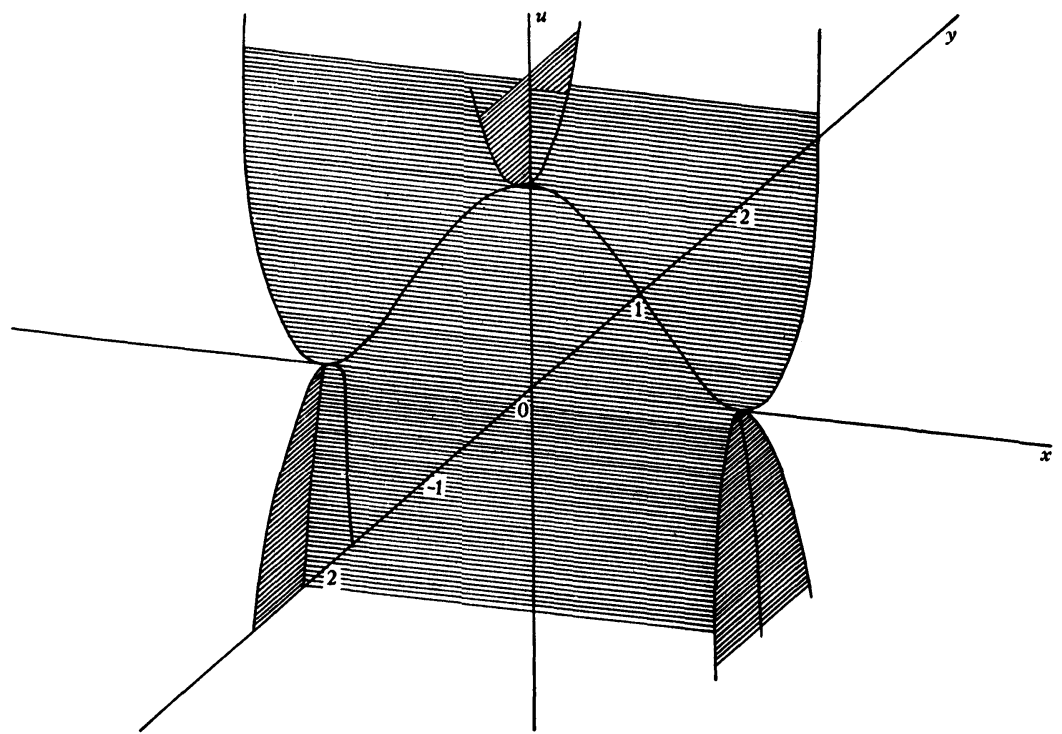

FIG. 17 
These hints are sufficient to characterize qualitatively the space curves for $a_{0} z^{n}+\cdots+a_{n}$, for the case when the total number of maxima, minima, and accessory points of inflection (each accessory point given a weight 2 ) is the largest possible.* Algebraically, this total number is obtained by a careful examination of $f^{\prime}(z)$ and $f^{\prime \prime}(z)$ for real $z$.

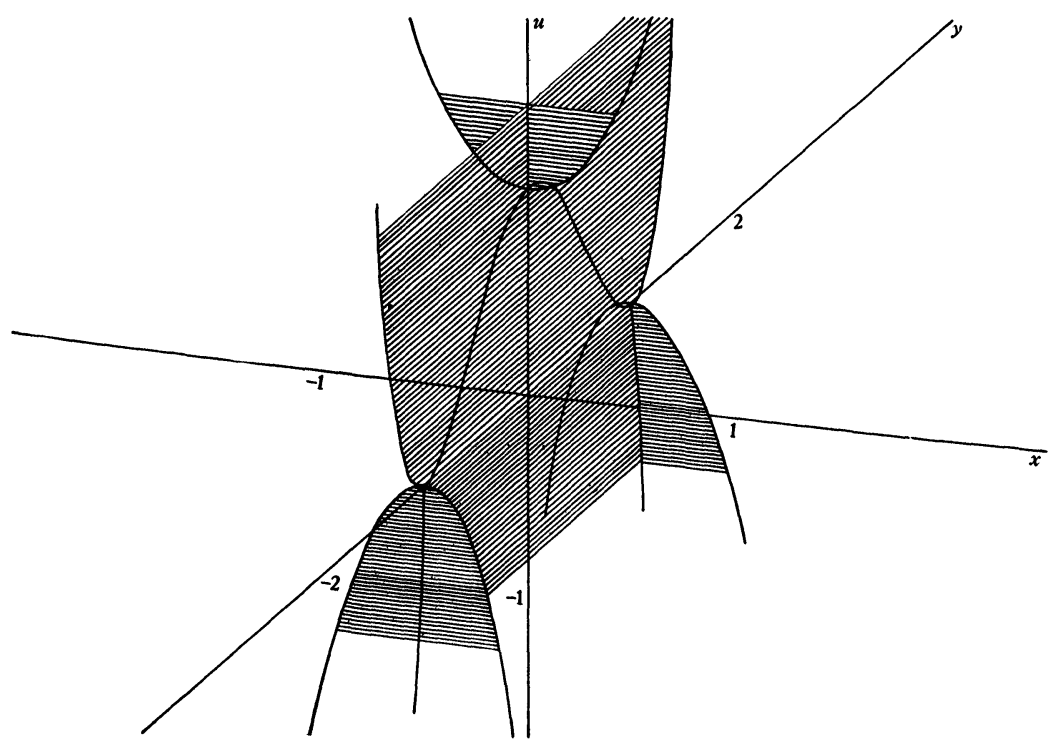

FIG. 18

(3) If our total number is less than $n-1$ (taking multiplicities into account), as in $z^{4}+2 z^{2}+1$, (one minimum, no points of inflection, and hence in particular no accessory points of inflection, total number $=1<3$ ), some of these free branches will in turn have coalesced. As an illustration of this state of affairs, we consider $z^{4}+2 z^{2}+1$. Since this function is obtained from $z^{4}$ $-2 z^{2}+1$ by a substitution $z=i z$, our whole space curve is obtained by rotating Fig. 17 through $\pi / 2$ around the $u$ axis, thus

* For a polynomial of degree $n$, remembering that each accessory point corresponds to one maximum and one minimum, we have $M_{a}+M_{i}+2 A \leqq n-1$, where $M_{a}=$ number of real maxima, $M_{i}=$ number of real minima, $A=$ number of accessory points. The sign of inequality holds when the curve does not possess the maximum number $n-1$ of extremes and some of the accessory points of inflection have become lost. 
changing the hyperbola in the $x y$ plane to one intersecting the $y$ axis.

If a continuous transformation of $z^{4}-2 z^{2}+1$ into $z^{4}+2 z^{2}+1$ is desired which shall illustrate in typical fashion how handles attached to the real curve $z^{4}-2 z^{2}+1$ first detach themselves from the real curve and are afterwards recaptured by it, a suitably chosen variation of coefficients passing through $z^{4}-z^{3}$ is preferable to our chain $z^{4}+\epsilon z^{2}, 2 \geqq \epsilon \geqq-2$. In the general case, the situation is governed by the curve $v(x, y)=0$; any point in the $x y$ plane where two branches of $v(x, y)=0$ intersect (double points of $v=0$ ) is the projection of a point on our space curve where two handles or detached branches are joined, and conversely. This agrees with the theory of conformal mapping and its breakdown at points for which $f^{\prime}(z)=0$. At all such points on the space curve where handles or branches are joined (including the real curve), they are of the character of intersections of a surface at a saddle point. If a point starts moving along any branch of the space curve at $u=+\infty$, it is always possible to travel along the space curve to $u=-\infty$. (This is a consequence of the last remark combined with the Fundamental Theorem.)

I hope that enough has been said about these space curves to justify the importance I attach to them for the problem of complex roots. A collection of strong wire models of such curves, and their introduction into advanced elementary work, is very desirable.

The University of Colorado 